\title{
Systematic Review \\ Exercise and Manual Therapy for Diabetic Peripheral Neuropathy: A Systematic Review
}

\author{
Mar Hernández-Secorún ${ }^{1,2}\left(\mathbb{D}\right.$, Concepción Vidal-Peracho ${ }^{1,3}$, Sergio Márquez-Gonzalvo ${ }^{1,2}$, \\ Jaime Corral-de-Toro ${ }^{1,2}$, Julián Müller-Thyssen-Uriarte ${ }^{1,2}$, Jacobo Rodríguez-Sanz ${ }^{4,5}$ (D), \\ María Orosia Lucha-López ${ }^{1,2, *(D)}$, José Miguel Tricás-Moreno ${ }^{1,2}$ and César Hidalgo-García ${ }^{1,2}$ (D)
}

1 Unity of Physiotherapy Research, Faculty of Health Sciences, University of Zaragoza, 50009 Zaragoza, Spain; marhsecorun@unizar.es (M.H.-S.); cvidal@unizar.es (C.V.-P.); 724250@unizar.es (S.M.-G.); 82825@unizar.es (J.C.-d.-T.); 732751@unizar.es (J.M.-T.-U.); jmtricas@unizar.es (J.M.T.-M.); hidalgo@unizar.es (C.H.-G.)

2 Faculty of Health Sciences, University of Zaragoza, 50009 Zaragoza, Spain

3 Department of Endocrinology and Nutrition, Hospital Royo Villanova SALUD, Barrio San Gregorio, s/n, 50015 Zaragoza, Spain

4 Faculty of Medicine and Health Sciences, Universitat Internacional de Catalunya, c/Josep Trueta s/n., 08195 Barcelona, Spain; jrodriguezs@uic.es

5 ACTIUM Research Group, 08195 Barcelona, Spain

* Correspondence: orolucha@unizar.es

Citation: Hernández-Secorún, M.; Vidal-Peracho, C.; MárquezGonzalvo, S.; Corral-de-Toro, J.; Müller-Thyssen-Uriarte, J.; Rodríguez-Sanz, J.; Lucha-López, M.O.; Tricás-Moreno, J.M.; Hidalgo-García, C. Exercise and Manual Therapy for Diabetic Peripheral Neuropathy: A Systematic Review. Appl. Sci. 2021, 11, 5665. https://doi.org/10.3390/ app11125665

Academic Editor: Zimi Sawacha

Received: 20 May 2021

Accepted: 16 June 2021

Published: 18 June 2021

Publisher's Note: MDPI stays neutra with regard to jurisdictional claims in published maps and institutional affiliations.

Copyright: (c) 2021 by the authors. Licensee MDPI, Basel, Switzerland. This article is an open access article distributed under the terms and conditions of the Creative Commons Attribution (CC BY) license (https:// creativecommons.org/licenses/by/ $4.0 /)$.

\begin{abstract}
Background: Diabetic peripheral neuropathy (DPN) is one of the most common complications of diabetes mellitus (DM). Control of hyperglycaemia as well as surgical decompression are effective treatments for these patients. However, surgery is not indicated for all candidates. Manual therapy and physical exercise have been shown to be effective for peripheral neuropathies, and exercise for DM. The aim is to review the effectiveness of manual therapy and/or exercise in patients with DPN. (2) Methods: Randomised controlled clinical trials comparing the effects of manual therapy and/or exercise on pain, function and/or balance were selected. The search strategy was performed in PubMed, PEDro, Scopus, Cochrane and Web of Science databases. The PRISMA statement was followed. (3) Results: A total of 656 articles were registered, and 29 were selected. There was little consensus on DPN criteria selection. Aerobic, strength and balance exercises are beneficial for DPN. Sessions of 30-60 min, three times per week for 8 weeks seems to be the most used dose. Manual therapy is effective in the short term. A combination of both modalities was more beneficial than alone in one study. (4) Conclusions: Exercise and manual therapy are beneficial for patients with DPN. More studies should be carried out for analysing the potential effect of combining manual therapy and exercise.
\end{abstract}

Keywords: diabetes mellitus; diabetic neuropathies; exercise; manual therapy

\section{Introduction}

Diabetes mellitus is a heterogeneous metabolic disease characterised by hyperglycaemia as a result of defects in insulin secretion, in insulin action or in both [1]. It is one of the most common chronic diseases, with an estimated prevalence of 366 million patients in 2030 [2]. Peripheral neuropathy is one of the most common complications of diabetic patients, with 25-50\% being affected, especially with type 2 diabetes [3-5]. Peripheral neuropathy is known as the injury of small- or large-diameter nerve fibres of the peripheral nervous system, resulting in altered motor, sensory, vibration and proprioception functions for large fibres, and pain, temperature and autonomic function for small fibres [6].

The pathophysiology of diabetic neuropathy is multifactorial, with influence from genetic, environmental, behavioural, metabolic, neurotrophic and even vascular factors [7]. The potential mechanisms of the nerve lesions in diabetes include hyperglycaemia (toxic/reactive metabolites stemming from the hyperglycaemia), microangiopathy and 
ischemia, cell signalling anomalies due to diacylglycerol and to protein kinase $\mathrm{C}$, sodium channel deregulation and demyelination [8]. Hyperglycaemia is the main factor of risk in the various types of diabetes, provoking lesions by microvascular and metabolic alterations. These vascular changes damage the primary sensory nerves through neuronal hypoxia and nutrient deficits [8,9]. The neural lesion occurs in both long and short fibres indiscriminately, with a differentiated clinical presentation in both cases. The most frequent alterations are produced in short fibres.

There is evidence that strict blood glucose control is effective in halting progression to diabetic neuropathy [10], as it controls the hyperglycaemic component of these patients. Invasive treatment, such as surgical decompression, can be useful for relieving neuropathic symptoms in some patients. However, not all diabetic patients with neuropathy are candidates for nerve decompression [4]. Conservative treatment such as exercising, receiving transcutaneous electrical nerve stimulation (TENS) and taking vitamin D supplements have been shown to be beneficial in reducing pain and improving function and balance in these patients [3,11,12].

During exercise, muscle contraction increases blood glucose capture to complement intramuscular glycogenolysis. To replenish glycogen stores, resting muscle captures glucose postprandially depending on circulating insulin. After exercise, both pathways increase glucose capture towards the muscle [13]. It has also been observed that exercise improves blood glucose control and quality of life, reduces cardiovascular risk and contributes to weight loss [14]. Consequently, exercise-based interventions are beneficial for patients with diabetic neuropathy.

Various systematic revisions have indicated that manual therapy is beneficial in improving function and symptoms in patients with peripheral neuropathies [15-17]. In a study with rats, Zhu et al. [4] observed the impact of these oscillations on diabetic neuropathy using mobilisations of the sciatic nerve. The authors found an attenuation of allodynia in the treatment area and a reduction in pro-inflammatory mediator concentration in the sciatic nerve branches.

In spite of the elevated prevalence of peripheral neuropathy and its major involvement in the morbidity of patients with diabetes, it is the chronic complication for which the least information is available about its pathogeny and epidemiology, and the one with the fewest standardised study methods. Additionally, no systematic review on its therapeutic management, including manual therapy and exercise, was found [9]. The aim of this study was to review the effectiveness of manual therapy and/or exercise in patients with diabetic neuropathy.

\section{Materials and Methods}

\subsection{Protocol and Registry}

A systematic review following the Preferred Reporting Items for Systematic Reviews and Meta-Analyses (PRISMA) [18] criteria was designed. This review was registered on the Open Science Framework digital platform with doi:10.17605/OSF.IO/3FS92 (https: / / osf.io/3fs92/, accessed on 3 March 2021).

\subsection{Search and Information Sources}

The search strategy followed the Population, Intervention, Comparison, Outcome and Study type (PICOS) method. Population was defined as individuals with diabetic neuropathy; Intervention, as manual therapy and/or exercise; and Comparison, as comparing with the control group and/or placebo and/or other conservative treatment. Outcome was found using outcomes related to balance, motor and sensory functions, and/or pain. Study type was limited to controlled clinical trials. The following databases were searched: PubMed, PEDro, Cochrane Library, Scopus and Web of Science. Various search strategies were used depending on the database and the filters permitted. The MESH terms used were "Diabetes Mellitus", "Diabetes Complications", "Peripheral Nervous System Diseases", "Diabetic Neuropathies", "Musculoskeletal Manipulations", “Therapy, Soft 
Tissue", "Manual therapy", "Physical Therapy", "Resistance Training" and "Exercise Therapy". The Boolean terms "OR" and "AND" were used to achieve a better search. Table 1 shows the search strategies used for each database. Search period was settled as between 26 November 2020 and 20 January 2021.

Table 1. Search strategy.

\begin{tabular}{|c|c|c|}
\hline Database & Search Strategy & Filters \\
\hline PubMed & $\begin{array}{l}\text { "Diabetes Mellitus" [Mesh] AND ("Diabetes } \\
\text { Complications" [Mesh] OR "Peripheral Nervous } \\
\text { System Diseases" [Mesh] OR "Diabetic } \\
\text { Neuropathies" [Mesh]) AND ("Musculoskeletal } \\
\text { Manipulations" [Mesh] OR “Therapy, Soft Tissue" } \\
\text { [Mesh] OR "Manual therapy" OR "Physical } \\
\text { Therapy" OR "Resistance Training" [Mesh] OR } \\
\text { "Exercise Therapy" [Mesh]) }\end{array}$ & $\begin{array}{ll}\text { - } & \text { Availability: Full text. } \\
\text { - } & \text { Article type: Clinical. Trial: Randomised } \\
\text { controlled trial. } \\
\text { - } \quad \text { Language: English, Spanish, French, Italian. }\end{array}$ \\
\hline Web of Science & $\begin{array}{l}\text { "Diabetes Mellitus" AND ("Diabetes Complications" } \\
\text { OR “Peripheral Nervous System Diseases" OR } \\
\text { "Diabetic Neuropathies") AND ("Musculoskeletal } \\
\text { Manipulations" OR “Therapy, Soft Tissue" OR } \\
\text { "Manual therapy" OR "Physical Therapy" OR } \\
\text { "Resistance Training" OR “Exercise Therapy") }\end{array}$ & $\begin{array}{ll}\text { - } & \text { Document type: Article. } \\
\text { - } & \text { Language: English, Spanish, French, Italian. }\end{array}$ \\
\hline SCOPUS & $\begin{array}{l}\text { "Diabetes Mellitus" AND ("Diabetes Complications" } \\
\text { OR "Peripheral Nervous System Diseases" OR } \\
\text { "Diabetic Neuropathies") AND ("Musculoskeletal } \\
\text { Manipulations" OR “Therapy, Soft Tissue" OR } \\
\text { "Manual therapy" OR “Physical Therapy" OR } \\
\text { "Resistance Training" OR "Exercise Therapy") AND } \\
\text { "Clinical Trial" }\end{array}$ & $\begin{array}{ll}\text { - } & \text { Document type: Article. } \\
\text { - } & \text { Language: English, Spanish, } \\
& \text { French, Italian. } \\
\text { - } & \text { Source type: Journal. }\end{array}$ \\
\hline Cochrane Library & $\begin{array}{l}\text { "Diabetes Mellitus" AND ("Diabetes Complications" } \\
\text { OR "Peripheral Nervous System Diseases" OR } \\
\text { "Diabetic Neuropathies") AND ("Musculoskeletal } \\
\text { Manipulations" OR “Therapy, Soft Tissue" OR } \\
\text { "Manual therapy" OR "Physical Therapy" OR } \\
\text { "Resistance Training" OR "Exercise Therapy") }\end{array}$ & $\begin{array}{ll}\text { - } & \text { Trials. } \\
\text { - } & \text { Language: English, Spanish, French, Italian. }\end{array}$ \\
\hline PEDro & "diabetic neuropathy" & $\begin{array}{l}\text { - Therapy: Fitness training (9), skills training } \\
\text { (6), strength training (2), "stretching, } \\
\text { mobilization, manipulation, massage" (2). } \\
\text { - Type publication: Clinical trial. } \\
\text { - } \quad \text { When searching: Match all search } \\
\text { terms (AND). }\end{array}$ \\
\hline
\end{tabular}

\subsection{Eligibility Criteria}

Inclusion criteria were: (1) controlled clinical trials that compared a manual therapy and/or exercise group with a control group and/or placebo group and/or other conservative treatment; (2) studies in which the sample included patients diagnosed with diabetic neuropathy; (3) studies that focused on pain, sensory and motor function, and balance outcomes; and (4) studies published in English, French, Italian or Spanish. Exclusion criteria were studies for which (1) surgery was contemplated as a selection and/or intervention process; or (2) another approach was used, except if it was in the context of comparing it with manual therapy or exercise techniques.

\subsection{Selection of Studies}

Two different authors (M.H.-S. and M.O.L.-L.) selected and extracted the data independently. They searched the references sources and screened the titles, abstracts and 
complete text, following the eligibility criteria. If there were any discrepancies in opinions or any doubts, a third author (C.H.-G.) was consulted to resolve them.

\subsection{Data Extraction Process}

For each study, the following information was extracted: (1) author and the year of publication; (2) sample definition; (3) inclusion criteria; (4) exclusion criteria; (5) groups; (6) intervention; (7) dose; (8) outcome; and (9) results.

\subsection{Risk of Bias in Individual Studies}

The Physiotherapy Evidence Database (PEDro) scale and Cochrane's Risk of Bias 2 (RoB 2) tool were used to evaluate the methodological quality of the clinical trials. The first instrument, the PEDro Scale, conforms to the Delphi list, based on the consensus of experts to help the reader to identify clinical trials with sufficient internal validity and statistical information to make their results interpretable. It consists of 11 criteria to answer, using "Yes" or "No". Each criteria fulfilled receives 1 point, if the information is clearly expressed in the study; the maximum score is 11 points [19].

The RoB 2 tool is Cochrane's second version for evaluating the risk of bias in clinical trials. The biases are assessed in 5 different domains, as follows: (1) randomisation process; (2) effect of assignment to the interventions; (3) data from the outcome; (4) outcome measurement; and (5) results reported. Within each domain, there are 1 or more questions to which to respond. The answers lead to judgements of "low risk of bias", "some concerns" or "high risk of bias" [20]. The tables and charts presenting the results obtained with RoB 2 were created using the risk-of-bias visualisation (robvis) tool [21].

\section{Results}

\subsection{Selection of Studies}

The literature search returned 656 articles (PubMed: 113, Web of Science: 5; Scopus: 466; PEDro: 19; Cochrane: 53). After eliminating duplicates, 566 articles remained for analysis.

The first analysis focused on article titles and abstracts, with 492 being excluded for study design, sample, lack of complete article and interventions other than manual therapy and/or exercise.

The 72 articles remaining were then analysed to select those that fulfilled the inclusion criteria. Of these, 29 articles were selected for analysis. The selection process is shown in a PRISMA diagram flow (Figure 1).

\subsection{Study Characteristics \\ 3.2.1. Sample}

The study characteristics and sample selection are described in Tables 2 and 3. All the articles offer a sample of 1476 patients. There were 14 articles that specified that the diabetes had to be type 2 [22-35]; the rest of the articles did not specify which type of diabetes should be selected for the sample.

Most of the articles included patients from 45 to 65 years old. There were 16 articles that included patients aged over 40 years [22,24-26,28,29,33-42]. In addition, six articles included patients older than 18 [23,43-47], while seven did not specify the age for the selection of their sample [27,30-32,48-50]. 


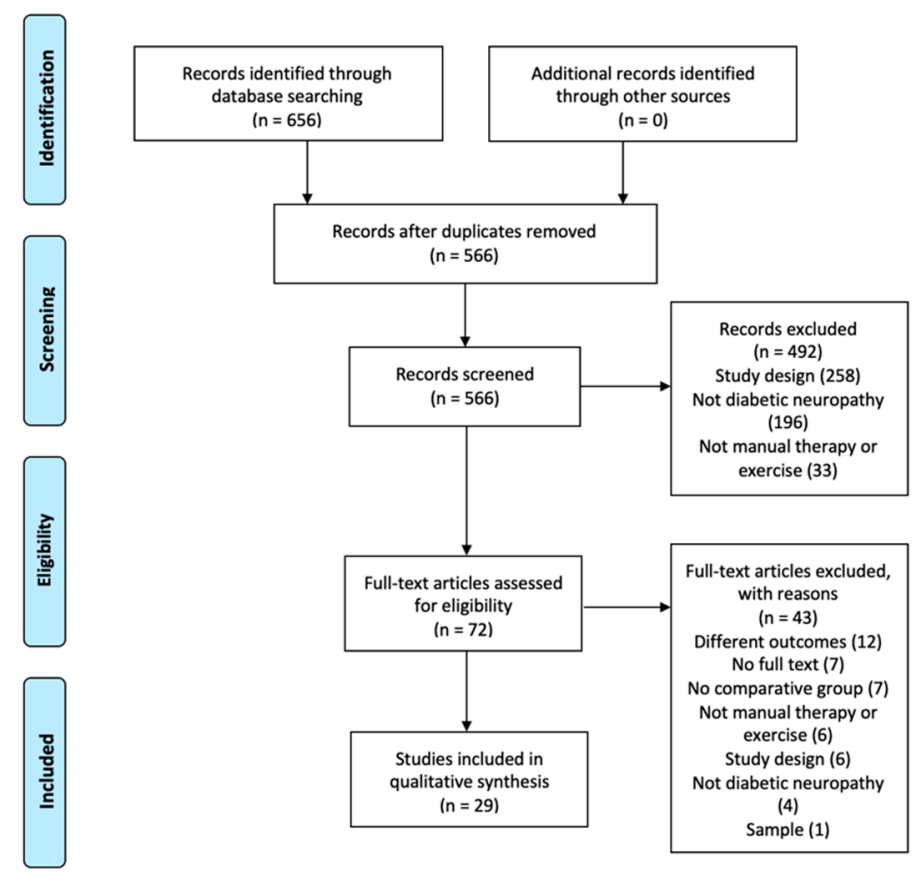

Figure 1. Flow diagram.

Blood analyses taking $\mathrm{HbA} 1 \mathrm{c}$ as a reference (cut-off value $\geq 6.5 \%$ ) and blood glucose levels (cut-off value $\geq 7.0 \mathrm{mmol} / \mathrm{L}$ or $\geq 120 \mathrm{mg} / \mathrm{dL}$ fasting value and $\geq 11.1 \mathrm{mmol} / \mathrm{L}$ or $\geq 200 \mathrm{mg} / \mathrm{dL}$ postprandial value); the Michigan questionnaire for neuropathies; and the American Diabetes Association criteria [50] were the criteria most used for the diagnosis of diabetic neuropathy. Three studies included an electrodiagnostic test to confirm the presence of neuropathy $[25,35,45]$. Other criteria observed in the different studies were physical examination tests, vibratory threshold, sensitivity to fine touch and physical skills.

Moreover, the typology of diabetic neuropathy is specific in a few articles, being described as peripheral neuropathy.

\subsubsection{Intervention}

There were 22 articles that included an intervention group with exercise [23,25-34,36$42,46,47,49,50]$ and six articles included an intervention group with manual therapy [22,24, 43-45,48], while one article combined both interventions [35].

\section{Exercise}

The majority of the studies compared an exercise group with a control group, implementing patient education or standard care. Four studies included three groups in their analysis: two intervention groups and one control group [23,34,37,47]. The intervention groups were mainly different proposals for types of exercise. The exception was Serry et al. [34], who compared exercise with TENS. Ten studies considered another type of training as a control group or considered two intervention groups directly [23,30-32,34,36,37,47]. 
Table 2. Selection criteria of the studies.

\begin{tabular}{|c|c|c|c|}
\hline Study & N (H/M) & Inclusion Criteria & Exclusion Criteria \\
\hline \multicolumn{4}{|c|}{ a. Exercise Intervention } \\
\hline Ahmad (2020) & $38(25 / 13)$ & $\begin{array}{ll}- & 45-75 \text { y. } \\
- & >7 \text { y of diabetes. } \\
- & \text { BMI 18,5-29,9 } \\
- & >2 / 13 \text { MSNI questionnaire (2 sympt. characteristic of DPN). } \\
\text { - } & >1 / 10 \text { MNSI ( } \downarrow \text { vibration, without plantar ulcers, without part or } \\
& \text { complete amputation, ability to walk short distances independently). }\end{array}$ & $\begin{array}{ll}\text { - } & \text { Other neurologic impairment. } \\
\text { - } & \text { Major vascular complication. } \\
\text { - } & \text { Severe retinopathy. } \\
\text { - } & \text { Severe nephropathy. } \\
\text { - } & \text { Severe lower limb msk impairment. } \\
\text { - } & \text { Not physiovascular implication. } \\
\text { Not therapy. }\end{array}$ \\
\hline Ahmad (2020) & $37(24 / 13)$ & $\begin{array}{ll}- & 45-75 \text { y. } \\
- & >7 \mathrm{y} \text { of diabetes. } \\
- & \text { BMI 18.5-29.9. } \\
- & >2 / 13 . \\
- & >2 / 13 \text { MNSI questionnaire. } \\
- & >1 / 10 \text { physical assessment }(\downarrow \text { sensorial vibration }) .\end{array}$ & $\begin{array}{ll}\text { - } & \text { Plantar ulcers. } \\
\text { - } & \text { Lower limb surgical and orthopaedic problems. } \\
\text { - } & \text { Other neurologic impairment. } \\
\text { - } & \text { Major vascular complication. } \\
\text { - } & \text { Severe retinopathy. } \\
& \text { Inability to walk independently with or without an } \\
\text { - } & \text { assistive device. } \\
\text { Not physical therapy. }\end{array}$ \\
\hline
\end{tabular}


Table 2. Cont.

$\begin{array}{lll}\text { Study } & \text { N }(\mathrm{H} / \mathrm{M}) & \text { Inclusion Criteria }\end{array}$

Foot ulcers.

Walking with assistive device.

Part or complete foot amputation.

Peripheral arterial disease.

Vision impairments.

- $\quad$ Neurologic or msk impairments.

- $\quad$ Acute sciatica.

- Vestibular dysfunction.

- Cognitive impairments.

- $\quad>30$ MDNS.

Known cardiac risks.

- Recent history of active retinal haemorrhage or a recent laser therapy $(<6 \mathrm{~m})$ for retinopathy.

- $\quad$ Recent vascularisation of coronary artery bypass grafting $(<3 \mathrm{~m})$.

- $\quad$ Seeking other therapies in DPN.

\begin{tabular}{|c|c|c|c|}
\hline Grewal (2015) & $35(16 / 19)$ & $\begin{array}{ll}\text { - } & 50-80 \mathrm{y} . \\
\text { - } & \text { Ability to walk independently for } 20 \mathrm{~m} . \\
\text { - } & \text { Medical diagnosis of type } 2 \text { diabetes. } \\
\text { - } & \text { DPN: criteria of American Diabetes Association statement, } \\
& \text { insensitivity to } 10 \mathrm{~g} \text { SWM, }<25 \text { V VPT. }\end{array}$ & $\begin{array}{ll}\text { - } & \text { Cognitive, vestibular or central neurological dysfunction. } \\
\text { - } & \text { Msk abnormality. } \\
\text { - } & \text { Active foot ulcers. } \\
\text { - } & \text { Charcot's joints. } \\
\text { - } & \text { History of a balance disorder unrelated to DPN. }\end{array}$ \\
\hline Jannu (2017) & $50(28 / 22)$ & $\begin{array}{ll}\text { - } & \text { Type } 2 \text { diabetes } 4-8 \text { y. } \\
\text { - } & \text { Lower limb muscle strength not less than grade } 3 . \\
\text { - } & \text { Moderate neuropathy: } 9-11 \text { Toronto scale. } \\
\text { - } & \text { BBS } 35-45 \text { score. } \\
\text { - } & \text { TUG }<15 \text { s. }\end{array}$ & $\begin{array}{ll}- & \text { Plantar ulcer and foot problems. } \\
- & \text { Any vestibular disorder. } \\
\text { - } & \text { History of any orthopaedic complications. } \\
\text { - } & \text { History of any neurological complications. } \\
\text { - } & \text { Any hearing and visual defects. }\end{array}$ \\
\hline
\end{tabular}


Table 2. Cont.

\begin{tabular}{|c|c|c|c|}
\hline Study & N (H/M) & Inclusion Criteria & Exclusion Criteria \\
\hline $\begin{array}{c}\text { Kanchanasamut } \\
\text { (2017) }\end{array}$ & $21(-)$ & $\begin{array}{ll}- & \text { Medical diagnosis of type } 2 \text { diabetes. } \\
\text { - } & \text { Loss of feeling. } \\
\text { - } & \text { BMI } 18-30 \mathrm{~kg} \cdot \mathrm{m}^{-2}\end{array}$ & $\begin{array}{ll}- & \text { Malignancy. } \\
- & \text { Myocardial infarction. } \\
- & \text { Stroke. } \\
- & \text { Hepatic failure. } \\
- & \text { Renal failure/dialysis. } \\
- & \text { Angina, embolism. } \\
- & \text { Cardiac arrhythmia. } \\
- & \text { Previous bypass surgery/angioplasty. } \\
- & \text { Foot/leg amputation. } \\
- & \text { Current or previous foot ulceration. } \\
- & \text { Reduced palpability of dorsalis pedis and tibialis } \\
- & \text { posterior arteries. } \\
\text { Participation in regular weight-bearing exercise. }\end{array}$ \\
\hline Kiani (2018) & $38(14 / 24)$ & $\begin{array}{ll}- & >40 \mathrm{y} . \\
- & >1 \mathrm{y} \text { diabetes. } \\
- & \text { Stable blood glucose control. }\end{array}$ & $\begin{array}{ll}\text { - } & \text { Foot ulceration. } \\
\text { - } & \text { Unstable heart disease. } \\
\text { - } & \text { Co-morbid conditions limiting exercise. } \\
\text { - } & \text { Any other disorder of the CNS causing weakness and } \\
& \text { sensory loss. }\end{array}$ \\
\hline Kuo (2019) & $38(21 / 17)$ & $\begin{array}{l}\text { - } \quad \text { Medical diagnose of type } 2 \text { diabetes: American Diabetes } \\
\text { Association criteria. } \\
\text { - }>26.5 \text { pinch strength. }\end{array}$ & $\begin{array}{l}\text { - } \quad \text { Neuro-msk disorders. } \\
\text { - } \quad \text { Traumatic nerve injuries of the upper limbs. } \\
\text { Trauma to the hand or congenital. anomalies of the wrist } \\
\text { and hand. } \\
\text { - } \quad \text { Skin infections or disease of the hands. } \\
\text { - } \quad \text { Cognitive deficits. } \\
\text { - } \quad<20 \text { y. }\end{array}$ \\
\hline Lee (2017) & $60(37 / 22)$ & $\begin{array}{ll}- & >65 \mathrm{y} . \\
\text { - } & \text { One or more falls within the past } 12 \text { months. } \\
\text { - } & \text { Physician's diagnosis of DPN. }\end{array}$ & $\begin{array}{ll}- & \text { Msk abnormalities. } \\
\text { - } & \text { Inability to stand independently. } \\
- & \text { Neurological impairment. } \\
- & <24 \text { Mini-Mental State Examination Score. }\end{array}$ \\
\hline
\end{tabular}


Table 2. Cont.

\begin{tabular}{|c|c|c|c|}
\hline Study & $\mathbf{N}(\mathrm{H} / \mathrm{M})$ & Inclusion Criteria & Exclusion Criteria \\
\hline Lee (2013) & $55(24 / 31)$ & $\begin{array}{l}\text { - } \quad>65 \mathrm{y} . \\
\text { Two or more falls during the previous } 12 \text { months } / 1 \text { fall }+>15 \mathrm{~s} \\
\text { TUG/recurrent unexplained falls. }\end{array}$ & $\begin{array}{ll}\text { - } & \text { Msk impairment (inability to walk independently). } \\
\text { - } & \text { Vision impairment. } \\
\text { - } & \text { Neurological impairment. } \\
\text { - } & \text { Vestibular diseases with diabetes-related etiology. } \\
\text { - } & \text { Dementia: }<24 \text { Mini-Mental. } \\
\text { - } & \text { Participation of }<80 \% \text { of the exercise program. } \\
\text { Unable to perform follow-up test. }\end{array}$ \\
\hline Mueller (2013) & $29(17 / 12)$ & $\begin{array}{l}\text { - } \quad \text { Type } 2 \text { diabetes. } \\
\text { - } \quad \text { PN: inability to sense } 5.07 \text { SWM in at least } 1 \text { spot of plantar foot, }<25 \\
\text { V VPT at plantar great toe. } \\
\text { - } \quad \text { Step count } 2000-9000 \text { steps } / \text { day. } \\
\text { - } \quad \text { Currently exercising }<3 \mathrm{t} / \mathrm{wk} \text {. } \\
\text { - } \quad<20 \mathrm{~min} / \mathrm{ss} \text {. }\end{array}$ & $\begin{array}{l}\text { - } \quad>138 \mathrm{~kg} . \\
\text { - } \quad \text { Severe foot deformity that requires custom } \\
\text { therapeutic footwear. } \\
\text { - } \quad \text { Comorbidity. } \\
\text { - } \quad \text { Medication that interferes with ability to exercise, } \\
\text { according to American Diabetes Association guidelines. }\end{array}$ \\
\hline $\begin{array}{l}\text { Nenkova } \\
\text { (2009) }\end{array}$ & $40(14 / 26)$ & $\begin{array}{l}\text { - } \quad 40-70 \text { y. } \\
\text { - } \quad \text { Type } 2 \text { diabetes. } \\
\text { - } \quad \text { Diffuse symmetrical sensory motor neuropathy. } \\
\text { - } \quad \text { Ability to walk without assistance or walking aid. } \\
\text { - } \quad \text { Strength of knee and ankle muscles grade } 3 \text { or greater by } \\
\text { manual testing. }\end{array}$ & 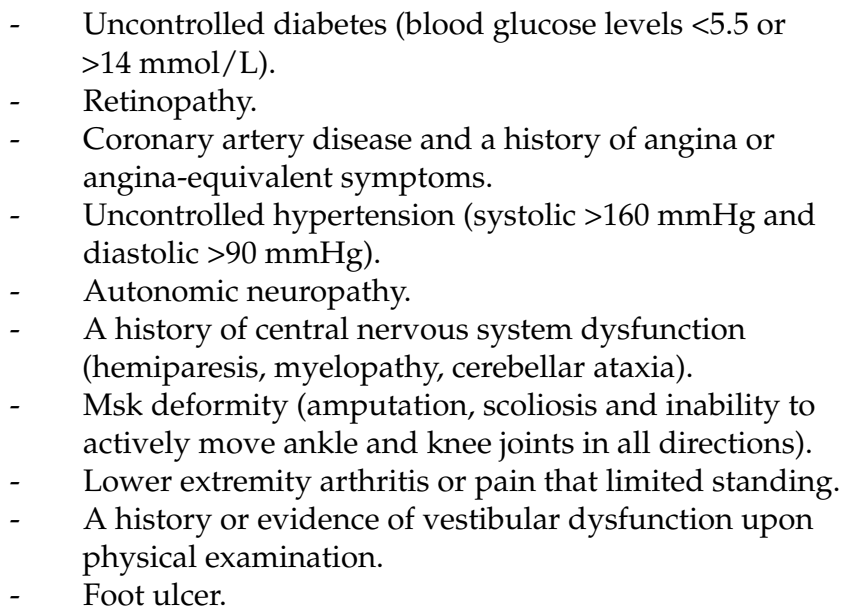 \\
\hline
\end{tabular}


Table 2. Cont.

\begin{tabular}{|c|c|c|c|}
\hline Study & $\mathbf{N}(\mathrm{H} / \mathrm{M})$ & Inclusion Criteria & Exclusion Criteria \\
\hline Quigley (2014) & $99(15 / 84)$ & $\begin{array}{l}\text { - } \\
\text { - } \quad \text { Able to ambulate household distances with or without an assistive } \\
\text { device, symptoms consistent with DSP (symmetric, distal lower } \\
\text { limb numbness or altered sensation). } \\
\text { PN: }>7 \text { MDNS. }\end{array}$ & $\begin{array}{ll}\text { - } & \text { Cognitive impairment: }<24 \text { Mini-Mental. } \\
\text { - } & \text { Severe disease: metastatic cancer, central } \\
\text { - } & \text { neurologic dysfunction. } \\
\text { - } & \text { Lower limb amputation. } \\
\text { - } & \text { Lower limb motor weakness (less than antigravity). } \\
& \text { Mobility limitation caused by altered lower extremity skin } \\
\text { - } \quad & \text { Medity/ulcer. } \\
\text { dyspnea at rest or minimal exertion, unstable angina). }\end{array}$ \\
\hline $\begin{array}{l}\text { Richardson } \\
\text { (2001) }\end{array}$ & $16(12 / 4)$ & $\begin{array}{l}\text { - } \quad 50-80 \mathrm{y} . \\
\text { - } \quad \text { Diabetes mellitus treated by diet, oral hypoglycaemic or insulin. } \\
\text { - } \quad \text { Lower extremity symptoms consistent with PN. } \\
\text { Ability to walk household distance without assistance or an } \\
\text { assistive device. } \\
\text { - Strength of ankle dorsiflexors, invertors and evertors at } \\
\text { least antigravity. } \\
\text { Conclusive electrodiagnostic evidence of a diffuse, primarily axonal, } \\
\text { peripheral polyneuropathy: sural response }(\mathrm{A}<6 \mathrm{yV} \text { with a normal } \\
\text { or minimally prolonged distal latency }(<5 \mathrm{~ms})) \text {; peroneal/tibial } \\
\text { responses }(\mathrm{A}<2 \mathrm{mV} \text { for peroneal and }<3 \mathrm{mV} \text { for tibial with a } \\
\text { normal distal latency }(<6.5 \mathrm{~ms})) .\end{array}$ & 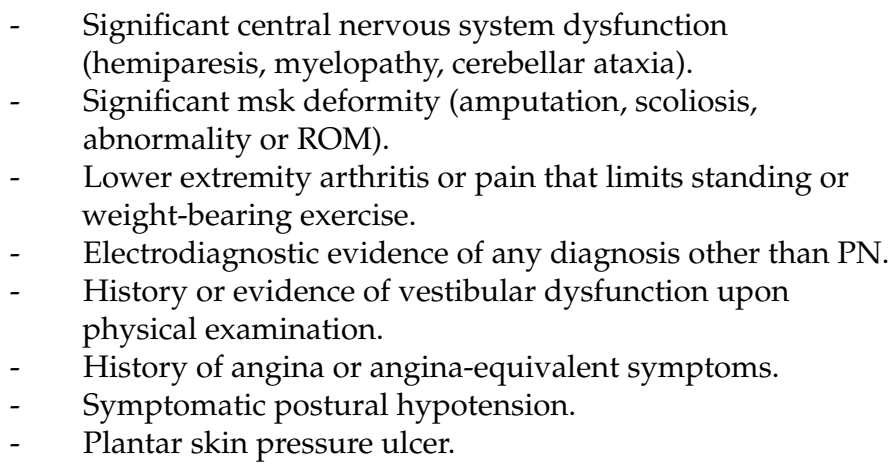 \\
\hline Serry (2016) & $60(28 / 32)$ & $\begin{array}{l}-\quad 45-60 \text { y. } \\
\text { - } \quad>10 \text { y type } 2 \text { diabetes mellitus. } \\
\text { - } \quad>5 \text { y DPN. } \\
\text { - } \quad \text { Ambulant and independent. } \\
\text { - } \quad \text { HbA1c }<6.5 \% \\
\text { - } \quad>4 \text { strength lower limb. } \\
\text { - } \quad \text { BMI } 18.5-29.9 \mathrm{~kg} \cdot \mathrm{m}^{-2}\end{array}$ & $\begin{array}{l}\text { - } \quad \text { Life-threatening diseases: renal failure, myocardial } \\
\text { infarction, heart failure. } \\
\text { - } \\
\text { Sensory manifestations due to other disease: lumbar } \\
\text { disc prolapsed. } \\
\text { - } \quad \text { Circulatory problems: intermittent claudications, skin } \\
\text { diseases or foot ulcers. } \\
\text { - } \quad \text { BMI }>30 \mathrm{~kg} \cdot \mathrm{m}^{-2} \text {. }\end{array}$ \\
\hline
\end{tabular}


Table 2. Cont.

\begin{tabular}{|c|c|c|c|}
\hline Study & N (H/M) & Inclusion Criteria & Exclusion Criteria \\
\hline $\begin{array}{l}\text { Seyedizadeh } \\
\quad(2020)\end{array}$ & $24(0 / 24)$ & $\begin{array}{ll}- & 45-60 \text { y. } \\
- & \text { DPN: MDNS and 10-point SWM. } \\
- & \text { Ability to carry out resistance and aerobic exercises. } \\
- & \text { Female menopause. }\end{array}$ & $\begin{array}{ll}\text { - } & \text { Amputation. } \\
\text { - } & \text { Sole injury. } \\
\text { - } & \text { Severe retinopathy, dialysis and neuropathy. } \\
\text { Upper body neuropathy or arthritis that could help reduce } \\
\text { and/or limit the pain. }\end{array}$ \\
\hline Song (2011) & $38(15 / 23)$ & $\begin{array}{ll}\text { - } & \text { Physician-diagnosed DPN. } \\
\text { - } & \text { Diabetes. }\end{array}$ & $\begin{array}{ll}\text { - } & \text { Msk impairments (inability to walk independently, lower } \\
\text { - } & \text { extremity strength grade 3, fracture or malformation). } \\
\text { - } & \text { Nevere osteoarthritis. } \\
\text { - } & \text { vestibular system. } \\
\text { - } & \text { Postural hypotension. } \\
\text { - } & \text { Psychiatric disorders. }\end{array}$ \\
\hline $\begin{array}{l}\text { Taveggia } \\
(2014)\end{array}$ & $27(10 / 17)$ & $\begin{array}{l}\text { - } \quad 45-90 \text { y. } \\
\text { - } \quad \text { DPN: clinical evaluation, diabetic neuropathy index criteria, SWM, } \\
\text { toe vibration). } \\
\text { - } \quad>3 \text { type } 2 \text { diabetes. } \\
\text { - } \quad \text { Walk autonomously. }\end{array}$ & $\begin{array}{ll}- & <5 \text { FIM locomotion scale. } \\
\text { - } & \text { Articular ankyloses, contractures, spasms with } \\
\text { - } & \text { locomotion effects. } \\
\text { - } & \text { Bone instability affecting lower limb functionality. } \\
& \text { Clinicopathologic conditions contraindicating } \\
\text { rehabilitation treatment. } \\
\text { - } \quad \text { Cutaneous lesions at lower limb. } \\
-\quad<22 \text { Mini-Mental. } \\
\text { - } \quad \text { Behavioural diseases involving aggressiveness or } \\
\text { psychotic disorders. }\end{array}$ \\
\hline Toth (2014) & $54(22 / 32)$ & 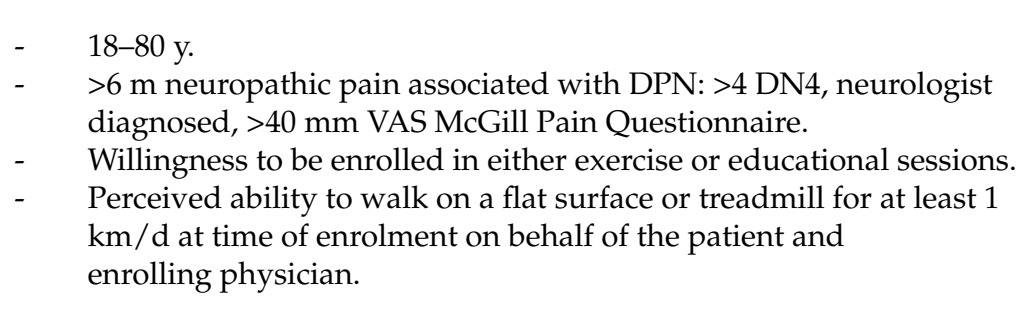 & $\begin{array}{l}\text { Another non-NeP source of pain that is more dominant } \\
\text { than the peripheral NeP or that cannot be } \\
\text { separated clinically. } \\
\text { - } \quad<6 \mathrm{~m} \mathrm{NeP.} \\
\text { - } \quad \text { Central nervous system cause of pain. } \\
\text { Absence of other health conditions: cardiovascular or } \\
\text { pulmonary disease, severe obesity, amputation, use of } \\
\text { mobility assistive devices or active neoplasia. }\end{array}$ \\
\hline
\end{tabular}


Table 2. Cont.

\begin{tabular}{|c|c|c|c|}
\hline Study & $\mathbf{N}(\mathbf{H} / \mathbf{M})$ & Inclusion Criteria & Exclusion Criteria \\
\hline $\begin{array}{c}\text { Venkataraman } \\
\text { (2019) }\end{array}$ & $143(-)$ & $\begin{array}{l}\text { - } \quad 40-79 \text { y. } \\
\text { DPN: } 25 \text { V neurothensiometer, positive monofilament test in }>2 \text { sites } \\
\text { of foot, }>7 \text { MDNS). } \\
\text { Type } 2 \text { diabetes. }\end{array}$ & $\begin{array}{ll}\text { - } & \text { Foot ulceration/infection/amputation. } \\
\text { - } & \text { Contraindication for physical activity or physiotherapy. } \\
\text { - } & \text { Non-diabetic neuropathy. } \\
\text { - } & \text { Nohol abuse. } \\
\text { Nurgical or medical conditions affecting functional mobility } \\
\text { and balance. }\end{array}$ \\
\hline \multicolumn{4}{|c|}{ b. Manual Therapy Intervention } \\
\hline $\begin{array}{l}\text { Chatchawan } \\
\text { (2015) }\end{array}$ & $60(20 / 40)$ & $\begin{array}{l}\text { - } \quad 40-70 \mathrm{y} \\
\text { - } \quad \text { DPN. } \\
\text { - } \quad \text { Impaired level of diabetic foot: peripheral sensory deficit SWMT on } \\
\text { 3rd and 5th toes, head of 1st and 3rd metatarsi; ability to walk } 10 \mathrm{~m} \\
\text { without walking aid. }\end{array}$ & $\begin{array}{ll}\text { - } & \text { Parkinson's disease and stroke. } \\
\text { - } & \text { Severe cognitive disability. } \\
\text { - } & \text { Acute illness, unstable hypertension and angina. } \\
\text { - } & \text { Myocardial infarction. } \\
\text { Fracture of the lower limb within } 6 \text { months before } \\
\text { - } \quad \text { Foot study. } \\
\text { - } \quad \text { Foot ulcer. } \\
\text { - } \quad \text { Dependence on alcohol/drugs. } \\
\text { - } \quad \text { Partial or complete blindness. }\end{array}$ \\
\hline Dalal (2014) & $58(31 / 27)$ & 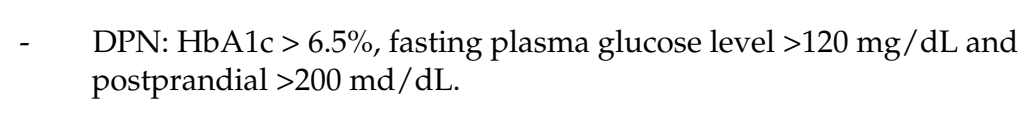 & $\begin{array}{l}\text { - End organ damage due to diabetes/any cause. } \\
\text { - Chronic disorders: malignancy, tuberculosis, asthma, } \\
\text { communicable disease. }\end{array}$ \\
\hline
\end{tabular}


Table 2. Cont.

\begin{tabular}{|c|c|c|c|}
\hline Study & N (H/M) & Inclusion Criteria & Exclusion Criteria \\
\hline $\begin{array}{l}\text { Gok Metin } \\
\text { (2017) }\end{array}$ & $46(11 / 35)$ & $\begin{array}{ll}- & 21-85 \mathrm{y} . \\
- & \mathrm{DP} 4>4 \mathrm{pt} . \\
- & \text { No history of other causes of } \mathrm{NeP} .\end{array}$ & $\begin{array}{ll}- & \text { Hand or foot wounds or previous related surgery. } \\
- & \text { Irritation. } \\
- & \text { Ulceration. } \\
- & \text { Soft tissue infection. } \\
- & \text { Essential oil allergies. } \\
- & \text { Blood coagulation disorders. } \\
- & \text { Pregnant. }\end{array}$ \\
\hline Talebi (2018) & $30(-)$ & $\begin{array}{l}\text { - } \quad 30-65 \text { y. } \\
\text { - } \quad \text { CTS diagnosed by a neurologist. } \\
\text { - } \quad \text { Complaint of pain and paresthesia in the distribution of median } \\
\text { nerve within the hand for at least } 6 \text { months. } \\
\text { - } \quad \text { Positive tinel sign and phalen sign. } \\
\text { - } \quad>2 \text { y diabetes. }\end{array}$ & $\begin{array}{ll}- & \text { History of carpal tunnel release. } \\
- & \text { Previous steroid injection. } \\
\text { - } & \text { Cervical radiculopathy. } \\
\text { - } & \text { Metabolic disorders other than diabetes. } \\
\text { - } & \text { Pregnancy. } \\
\text { - } & \text { History of neck/shoulder or arm trauma and atrophy of } \\
& \text { thenar muscles. }\end{array}$ \\
\hline Xie (2019) & $119(63 / 56)$ & $\begin{array}{l}\text { - } \quad \text { 18-80 y. } \\
\text { - } \quad \text { Type } 1 \text { or } 2 \text { diabetes. } \\
\text { - } \quad \text { Primary diagnosis of DPN: impaired light touch by 10g SWMT, } \\
\text { VPT }>16 \text { V each foot, }>6 \text { Toronto Clinical Scoring System. } \\
\text { - No foot ulcers or active signs of skin disease. }\end{array}$ & $\begin{array}{ll}\text { - } & \text { Cardiovascular or mental illness. } \\
\text { - } & \text { Non-diabetic peripheral nephropathy. } \\
\text { - } & \text { Ketacidosis or hyperosmolar coma. }\end{array}$ \\
\hline
\end{tabular}


Table 2. Cont.

\begin{tabular}{|c|c|c|c|c|c|c|c|c|c|}
\hline Study & \multicolumn{2}{|c|}{ N (H/M) } & \multicolumn{4}{|c|}{ Inclusion Criteria } & \multicolumn{3}{|c|}{ Exclusion Criteria } \\
\hline \multicolumn{10}{|c|}{ c. Combinate intervention } \\
\hline $\begin{array}{c}\text { Shourabi } \\
(2020)\end{array}$ & \multicolumn{6}{|c|}{$\begin{array}{ll}\text { - } & 38-58 \mathrm{y} \\
\text { - } & \text { Type } 2 \text { diabetes. } \\
\text { - } & \text { DPN. } \\
\text { - } & \text { Sedentary + able to walk } 1-6 \mathrm{~km} \text { distance without assistance. } \\
\text { - } & \text { Medication of metformin }(500 \mathrm{mg}, 2 \mathrm{t} / \mathrm{d})+\text { glibenclamide }(5-10 \mathrm{mg} \\
& \text { fasted state). }\end{array}$} & $\begin{array}{l}- \\
- \\
- \\
- \\
-\end{array}$ & \multicolumn{2}{|c|}{$\begin{array}{l}\text { Central nervous system dysfunction. } \\
\text { Significant msk deformity or pain that limits exercise. } \\
\text { Severe cardiovascular diseases. } \\
\text { Vestibular dysfunction. } \\
\text { Angina-equivalent symptoms. } \\
\text { Plantar skin pressure ulcers. }\end{array}$} \\
\hline \multicolumn{10}{|c|}{$\begin{array}{l}\text { BMI: body mass index; CTS: carpal tunnel syndrome; DPN: diabetic peripheral neuropathy; FIM: functional independence measure; MDNS: Michigan Diabetes Neuropathy Score; mg: milligrams; MNSI: } \\
\text { Michigan Neuropathy Screening Instrument; msk: musculoskeletal; NeP: neuropathic pain; ss: sessions; s: seconds; SWM: Semmes-Weinstein monofilament; PN: peripheral neuropathy; VPT: vibration } \\
\text { perception threshold; y: years. }\end{array}$} \\
\hline Study & Groups & & Dose & Description & & Outcomes & & Results & \\
\hline \multicolumn{10}{|c|}{ a. Exercise intervention } \\
\hline Ahmad (2020) & $\begin{array}{l}\text { G1: Intervention- } \\
\text { Exercise. } \\
\text { G2: Control. }\end{array}$ & & $\begin{array}{l}\text { Exercise: } 24 \\
\text { ss.3t/wk·8 wk } \\
\text { (rest } 48 \mathrm{~h}) . \\
\text { Control: } \mathrm{G} 1+\mathrm{G} 2 \text {. } \\
30 \mathrm{~min} \cdot 1 \mathrm{t} \cdot 2 \mathrm{wk} \text {. }\end{array}$ & $\begin{array}{l}\text { Exercise: } \\
\text { Sensoriomotor + gait } \\
\text { pattern. } 10 \mathrm{~min} \\
\text { warm-up }+50-60 \mathrm{~min} \\
\text { train }+5-10 \mathrm{~min} \\
\text { cold-down. } \\
\text { Control: Education. }\end{array}$ & & $\begin{array}{l}\text { Propioception: front, back, } \\
\text { left, right } \\
\text { Latency, amplitude, duration } \\
\text { NCV of peroneal and tibial ner } \\
\% \text { MVIC open, close eyes, } \\
\text { treadmill walking: TA, MG, } \\
\text { VL, MF } \\
\text { Co-contraction index: stand EC } \\
\text { EC, gait }\end{array}$ & Orve & $\begin{array}{l}\text { G1: }+\mathrm{NCV} \text { peroneal/tibial; } \\
\text {-Distal tibial latency. EO: TA, MG, } \\
\text { MF t.gp }(p<0.05) \cdot \downarrow \mathrm{MF} / \uparrow \mathrm{TA} \\
\text { treadmill. Co-contraction }(p<0.05) \text {. } \\
\text { G2: +NCV tibial, +DL tibial. } \\
\uparrow \mathrm{TA}-\mathrm{MF} \text { treadmill }(p<0.05) \text {. } \\
\text { G1 vs. G2: Significant difference in } \\
\text { all proprioception angles for G1. }\end{array}$ & $\begin{array}{l}\text { Balance: Improvement in } \\
\text { both groups, better for G1. } \\
\text { Function: Not measured. } \\
\text { Pain: Not measured. }\end{array}$ \\
\hline Ahmad (2020) & $\begin{array}{l}\text { G1: Intervention- } \\
\text { Exercise. } \\
\text { G2: Control. }\end{array}$ & & $\begin{array}{l}\text { Exercise: } 3 \mathrm{t} / \\
\text { wk } 8 \mathrm{wk} . \\
\text { Control: } \mathrm{G} 1+\mathrm{G} 2 . \\
30 \mathrm{~min} \cdot 1 \mathrm{t} \cdot 2 \mathrm{wk} .\end{array}$ & $\begin{array}{l}\text { Exercise: Core }+ \\
\text { balance + gait pattern } \\
10 \text { min warm-up }+ \\
\text { 50-60 min train }+ \\
\text { 5-10 min cold-down. } \\
\text { Control: Education. }\end{array}$ & & $\begin{array}{l}\text { FRT } \\
\text { TUG } \\
\text { OLS: EO, EC right; EO, EC left } \\
\text { COP range: front, back left, rig } \\
\text { COP sway VF: F-B, L-R } \\
\text { COP sway WVF: F-B, L-R } \\
\text { Propioception: front, back } \\
\text { left, right }\end{array}$ & & $\begin{array}{l}\text { G1 vs. G2: COP range + } \\
\text { Proprioception front for G1 } \\
(p<0.05) \text {. Significantly different } t \\
\text { effect for all outcomes except COP } \\
\text { sway VF F-B, OLS EO right. } \\
\text { Effect-age for OLS EO-EC left, EC } \\
\text { right, COP sway WVF F-B. } \\
\text { Group-affects all outcomes except } \\
\text { COP sway. T effect—age for OLS } \\
\text { EO-EC left and EC right. }(p<0.05) \text {. }\end{array}$ & $\begin{array}{l}\text { Balance: Improvement in } \\
\text { both groups, better for G1. } \\
\text { Function: No } \\
\text { improvement. } \\
\text { Pain: Not measured. }\end{array}$ \\
\hline
\end{tabular}


Table 3. Cont.

\begin{tabular}{|c|c|c|c|c|c|c|}
\hline Study & Groups & Dose & Description & Outcomes & Results & \\
\hline Cox (2020) & $\begin{array}{l}\text { G1: Intervention- } \\
\text { Exercise C-MICT. } \\
\text { G2: Intervention- } \\
\text { Exercise C-HIIT. } \\
\text { G3: Control. }\end{array}$ & $\begin{array}{ll} & \\
\text { C-MICT: } \\
210 \mathrm{~min} / \mathrm{wk} \cdot 4 \mathrm{t} / \\
& \mathrm{wk} \cdot 8 \mathrm{wk} . \\
\text { C-HIIT: } \\
\text { 78 min } / \mathrm{wk} \cdot 3 \mathrm{t} / \mathrm{sem} \cdot 8 \mathrm{wl} \\
\text { Control: } \mathrm{G} 2.8 \mathrm{wk} .\end{array}$ & $\begin{array}{ll}\text { - } & \text { C-MICT: } 2 \text { ss aerobic } \\
& \text { (55-69\% HRpeak)+ } \\
& \text { resistance (I moderate); } \\
& 2 \text { ss aerobic (55-69\% } \\
& \text { HRpeak). } \\
- & \text { C-HIIT: } 3 \text { min } \cdot 50-60 \% \\
\text { k. } \quad \text { HRpeak }+ \\
\\
4 \text { min } \cdot 85-95 \% \text { HRpeak } \\
+8 \cdot 1 \text { min RPE }>7 . \\
\text { - Control: Care. }\end{array}$ & $\begin{array}{ll}- & \text { NMQ } \\
- & \text { NTSS-6 } \\
- & \text { QST } \\
- & \text { Adverse effect } \\
- & \text { IPAQ } \\
- & \text { HbA1c + fasting glucose }\end{array}$ & $\begin{array}{l}\text { G1: } 96.5 \% \text { adherence. } \\
\text { G2: } 97.9 \% \text { adherence. } \\
\text { G1 vs. G3: Significant difference in } \\
\text { pain intensity for G1 }(p<0.05) \text {. } \\
\text { G2 vs. G3: Significant difference in } \\
\text { pain intensity for G2 }(p<0.05) \text {. } \\
\text { Adverse effect: C-HIIT } \uparrow \text { risk of } \\
\text { adverse events and msk for } \\
100 \text { h train. }\end{array}$ & $\begin{array}{l}\text { Balance: Not measured. } \\
\text { Function: No } \\
\text { improvement. } \\
\text { Pain: Better results for } \\
\text { intervention groups. }\end{array}$ \\
\hline Dixit (2016) & $\begin{array}{l}\text { G1: Intervention- } \\
\text { Exercise. } \\
\text { G2: Control. }\end{array}$ & $\begin{array}{l}\text { Exercise: } 3-6 \mathrm{t} / \\
\text { wk. } 8 \mathrm{wk} . \\
\text { Control: } \mathrm{G} 1+\mathrm{G} 2.1 \\
\mathrm{t} \cdot 2 \mathrm{wk} \cdot 8 \mathrm{wk} .\end{array}$ & $\begin{array}{l}\text { Exercise: I moderate, } \\
\text { between } 150 \text { and } \\
360 \mathrm{~min} / \mathrm{wk} . \\
\text { Control: Phone calls. } \\
\text { Standard medical care }+ \\
\text { education on foot care } \\
\text { and diet. }\end{array}$ & $\begin{array}{l}\text { - } \quad \text { EO+ EC: } x \text {-axis, y -axis, VM, } \\
\text { AP, ML. } \\
\text { - } \quad \text { EOF+ ECF: } x \text {-axis, y -axis, VM, } \\
\text { AP, ML. } \\
\text { - Waist circumference. }\end{array}$ & $\begin{array}{l}\text { G1 vs. G2: Significant difference in } \\
\text { oscillatory velocity ECF } x \text {-axis and } \\
\text { EOF ML }(p<0.05) \text { for G1. }\end{array}$ & $\begin{array}{l}\text { Balance: Greater } \\
\text { improvement in G1. } \\
\text { Function: Not measured. } \\
\text { Pain: Not measured. }\end{array}$ \\
\hline Grewal (2015) & $\begin{array}{l}\text { G1: Intervention- } \\
\text { Exercise. } \\
\text { G2: Control. }\end{array}$ & $\begin{array}{l}\text { Exercise: } 6 \cdot 20 \\
\text { rep. } 45 \mathrm{~min} \cdot 2 \mathrm{t} / \mathrm{wk} \cdot 4 \\
\text { wk. } \\
\text { Control: } \mathrm{G} 2.8 \mathrm{wk} .\end{array}$ & $\begin{array}{l}\text { Exercise: point-to-point } \\
\text { ankle-reaching task and } \\
\text { a virtual obstacle } \\
\text { crossing. } \\
\text { Control: Standard } \\
\text { medical care. }\end{array}$ & $\begin{array}{l}\text { - } \quad \text { EO + EC: CoM sway, CoM AP } \\
\text { sway, CoM ML sway, ankle sway, } \\
\text { hip sway. } \\
\text { SF-12. } \\
\text { - } \quad \text { Daily physical activities } \\
\text { monitored during } 48 \text { h: time spent } \\
\text { sitting, standing, walking, } \\
\text { total steps. }\end{array}$ & $\begin{array}{l}\text { G1: }>26.09 \% \text { improvement in } \\
\text { balance outcomes. Between } \\
-0.04 / 27.68 \% \text { of change for SF-12 } \\
\text { and ADL. } \\
\text { G2: Between }-34.29 / 23.03 \% \text { of } \\
\text { change for outcomes. } \\
\text { G1 vs. G2: Significant difference in } \\
\text { EO (not CoM AP sway), EC ankle } \\
\text { sway, SF-12 mental component } \\
\text { for G1. }(p<0.05) \text {. }\end{array}$ & $\begin{array}{l}\text { Balance: Improvement in } \\
\text { both groups. Better for G1. } \\
\text { Function: Improvement } \\
\text { in both groups. Better } \\
\text { for G1. } \\
\text { Pain: Not measured. }\end{array}$ \\
\hline Jannu (2017) & $\begin{array}{l}\text { G1: Intervention- } \\
\text { Exercise WooB. } \\
\text { G2: Intervention- } \\
\text { Exercise } \\
\text { ST. }\end{array}$ & $\begin{array}{ll}\text { - } & \text { WooB: } 45 \mathrm{~min} \cdot 8 \mathrm{wk} \\
\text { - } & \text { ST: } 45 \mathrm{~min} \cdot 8 \mathrm{wk} .\end{array}$ & $\begin{array}{l}\text { WooB: } 30 \mathrm{~min} \\
\text { conventional } \\
\text { physiotherapy } \\
+15 \mathrm{~min} \mathrm{WB.} \\
\text { ST: } 30 \mathrm{~min} \\
\text { conventional } \\
\text { physiotherapy } \\
+15 \text { min ST. }\end{array}$ & $\begin{array}{ll}- & \text { BBS. } \\
- & \text { TUG. }\end{array}$ & $\begin{array}{l}\text { G1: No significant differences } \\
(p>0.05) \text {. } \\
\text { G2: Significant differences }(p<0.05) \\
\text { for BBS/TUG. } \\
\text { G1 vs. G2: Significant difference for } \\
\text { G2 }(p<0.05) \text {. }\end{array}$ & $\begin{array}{l}\text { Balance: Better for G2. } \\
\text { Function: Better for G2. } \\
\text { Pain: Not measured. }\end{array}$ \\
\hline
\end{tabular}


Table 3. Cont.

\begin{tabular}{|c|c|c|c|c|c|c|}
\hline Study & Groups & Dose & Description & Outcomes & Results & \\
\hline $\begin{array}{l}\text { Kanchanasamut } \\
\text { (2017) }\end{array}$ & $\begin{array}{l}\text { G1: Intervention- } \\
\text { Exercise. } \\
\text { G2: Control. }\end{array}$ & $\begin{array}{l}\text { Exercise: } 5 \mathrm{t} / \mathrm{wk} \cdot 2 \mathrm{wk} \\
(\text { each level) } 8 \text { wk. } \\
\text { Control: } \mathrm{G} 1+\mathrm{G} 2 . \\
8 \mathrm{wk} .\end{array}$ & $\begin{array}{l}\text { Exercise: } \\
\text { weight-bearing exercise } \\
\text { on a mini-trampoline. } \\
5 \text { min warm-up }+ \\
10 \cdot 10 \mathrm{~s}+5 \text { min } \\
\text { cold-down. } \\
\quad \text { Control: } \text { Foot care. }\end{array}$ & $\begin{array}{l}\text { - } \quad \text { ROM: flexion, extension 1st MTP } \\
\text { right and left. } \\
\text { Peak plantar pressure: hallux, } \\
\text { medial and lateral forefoot, } \\
\text { midfoot, heel. Right and left. }\end{array}$ & $\begin{array}{l}\text { G1: Significant improvement } \\
(p<0.05) \text { flex-Ext } 1 \text { st MTP }(0-8 \mathrm{wk} / \\
0-20 \mathrm{wk}) \text {; peak plantar pressure } \\
\text { lateral left, medial right forefoot } \\
(0-20 \mathrm{wk} / 8-20 \mathrm{wk}) \text {; flex } 1 \text { st MTP } \\
\text { left }(8-20 \mathrm{wk}) \text {. } \\
\text { G2: No significant improvement } \\
(p>0.05) \text {. } \\
\text { G1 ws. G2: Significant difference in } \\
\text { flex } 1 \text { st MTP ( } 20 \mathrm{wk}) \text {, ext } 1 \text { st MTP } \\
\text { left ( } 20 \mathrm{wk}), \text { pressure perception left } \\
(8 \mathrm{wk}) \text { and vibration left }(8-20 \mathrm{wk}) \\
\text { and right }(20 \mathrm{wk}) \text { for G1 }(p<0.05) .\end{array}$ & $\begin{array}{l}\text { Balance: Better for G1 at } \\
8 \text { and } 20 \mathrm{wk} \text {. } \\
\text { Function: Better for G1 at } \\
20 \mathrm{wk} \text {. } \\
\text { Pain: Not measured. }\end{array}$ \\
\hline Kiani (2018) & $\begin{array}{l}\text { G1: Intervention- } \\
\text { Aerobic. } \\
\text { G2: Intervention- } \\
\text { Balance. }\end{array}$ & $\begin{array}{l}\text { - } \quad \text { Aerobic: } 3 \mathrm{t} / \\
\text { wk } 6 \mathrm{wk} . \\
\text { Balance: } \mathrm{G} 2 . \\
30 \mathrm{~min} \cdot 3 \mathrm{t} / \mathrm{wk} \cdot 6 \mathrm{wk} .\end{array}$ & 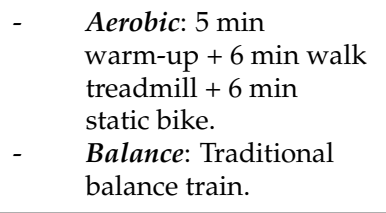 & $\begin{array}{ll}- & \text { FRT. } \\
- & \text { BRT. } \\
- & \text { BBT. }\end{array}$ & $\begin{array}{l}\text { G1 vs. G2: Significant difference in } \\
\text { BBT ( } 6 \mathrm{wk}) \text {, FRT and BRT ( } 3 \mathrm{wk} / \\
6 \mathrm{wk}) \text { for G1. }\end{array}$ & $\begin{array}{l}\text { Balance: Better for G1 at } \\
3 \text { and } 6 \text { wk. } \\
\text { Function: Better for G1 at } \\
3 \text { and } 6 \text { wk. } \\
\text { Pain: Not measured. }\end{array}$ \\
\hline Kuo (2019) & $\begin{array}{l}\text { G1: Intervention- } \\
\text { Biofeedback. } \\
\text { G2: Intervention- } \\
\text { Multimodal. }\end{array}$ & $\begin{array}{ll}\text { - } & \text { Biofeedback: } \\
30 \mathrm{~min} \cdot 2 \mathrm{t} / \mathrm{wk} \cdot 6 \mathrm{wk} . \\
\text { - } \quad \text { Multimodal: } \mathrm{G} 2 . \\
30 \mathrm{~min} \cdot 2 \mathrm{t} / \mathrm{wk} \cdot 6 \mathrm{wk} .\end{array}$ & $\begin{array}{l}\text { Biofeedback: } \\
\text { Biofeedback protocol. } \\
\text { Multimodal: } \\
\text { home-based tendon } \\
\text { gliding + resistance } \\
\text { train + diabetes care. }\end{array}$ & $\begin{array}{ll}- & \text { Purdue Pegboard Test. } \\
- & \text { S2PD. } \\
- & \text { M2PD. } \\
- & \text { FPpeak. } \\
- & \text { Force ratio. } \\
- & \text { Maximum pinch strength. } \\
- & \text { \% of maximum pinch strength. } \\
- & \text { MHQ scale. } \\
- & \text { Diabetes-39. }\end{array}$ & $\begin{array}{l}\text { G1 vs. G2: Significant difference in } \\
\text { S2PD, D2PD, \% and maximum } \\
\text { pinch strength, Purdue Pegboard } \\
\text { Test, Diabetes-39 (control, sexual } \\
\text { function, energy and mobility) } \\
\text { for G1. }\end{array}$ & $\begin{array}{l}\text { Balance: Not measured. } \\
\text { Function: Better for G1. } \\
\text { Pain: Not measured. }\end{array}$ \\
\hline Lee (2017) & $\begin{array}{l}\text { G1: Intervention- } \\
\text { Vibratory } \\
\text { exercise. } \\
\text { G2: Intervention- } \\
\text { Strength } \\
\text { exercise. }\end{array}$ & $\begin{array}{l}\text { Vibratory: } \\
11 \mathrm{~min} \cdot 3 \mathrm{t} / \mathrm{wk} \cdot 6 \mathrm{wk} . \\
\text { Strength: } \mathrm{G} 2 . \\
11 \mathrm{~min} \cdot 3 \mathrm{t} / \mathrm{wk} \cdot 6 \mathrm{wk} .\end{array}$ & $\begin{array}{l}\text { Vibratory: Vibratory } \\
\text { training. } \\
\text { - Strength: Lower limb } \\
\text { training. }\end{array}$ & $\begin{array}{ll}- & \text { CPT. } \\
- & \text { HPT. } \\
- & \text { VPT. }\end{array}$ & $\begin{array}{l}\text { G1: Significant improvement } \\
(p<0.05) \text { in VPT } \\
\text { G2: No significant improvement } \\
(p>0.05) \text {. } \\
\text { G1 vs. G2: Significant differences } \\
(p<0.05) \text { in VPT for G1. }\end{array}$ & $\begin{array}{l}\text { Balance: Not measured. } \\
\text { Function: Improvement } \\
\text { in G1. Better for G1. } \\
\text { Pain: Not measured. }\end{array}$ \\
\hline
\end{tabular}


Table 3. Cont.

\begin{tabular}{|c|c|c|c|c|c|c|}
\hline Study & Groups & Dose & Description & Outcomes & Results & \\
\hline Lee (2013) & $\begin{array}{l}\text { G1: Intervention- } \\
\text { Exercise WBV. } \\
\text { G2: Intervention- } \\
\text { Exercise BE2. } \\
\text { G3: Control. }\end{array}$ & $\begin{array}{ll}- & W B V: \\
& 60 \mathrm{~min} \cdot 2 \mathrm{t} / \mathrm{wk} \cdot 6 \mathrm{wk} . \\
& \text { BE: } \\
& 60 \mathrm{~min} \cdot 2 \mathrm{t} / \mathrm{wk} \cdot 6 \mathrm{wk} . \\
& \text { Control: } 6 \mathrm{wk} .\end{array}$ & $\begin{array}{l}\text { - } \quad \text { WBV: } 10 \text { min warm-up } \\
\quad+40 \text { min } \\
\text { balance/vibratory + } \\
10 \text { min cold-down. } \\
\text { BE: } 10 \text { min warm-up + } \\
40 \text { min balance + } \\
\text { 10 min cold-down. } \\
\quad \text { Control: - }\end{array}$ & $\begin{array}{ll}- & \text { HbA1c. } \\
- & \text { EO and EC: AP, ML, VM. } \\
- & \text { BBS. } \\
- & \text { FRT. } \\
- & \text { TUG. } \\
- & \text { FTSTS. }\end{array}$ & $\begin{array}{l}\text { G1: Significant improvement } \\
(p<0.05) \text { in HbA1c, postural sway, } \\
\text { OLS, FRT, BBS, TUG, FTSTS. } \\
\text { G2: Significant improvement } \\
(p<0.05) \text { in postural sway, OLS, FRT, } \\
\text { BBS, TUG, FTSTS. } \\
\text { G3: No significant improvement } \\
(p>0.05) \\
\text { G1 vs. G2: Significant differences } \\
(p<0.05) \text { in HbA1c, postural sway, } \\
\text { OLS, BBS, TUG, FTSTS for G1. } \\
\text { G1 vs. G3: Significant differences } \\
(p<0.05) \text { in OLS for G1. } \\
\text { G2 vs. G3: Significant differences } \\
(p<0.05) \text { in OLS for G2. }\end{array}$ & $\begin{array}{l}\text { Balance: Improvement in } \\
\text { G1 and G2. Better for G1 } \\
\text { vs. G2 and G3; of G2 vs. } \\
\text { G3. } \\
\text { Function: Improvement } \\
\text { in G1 and G2. Better for } \\
\text { G1 vs. G2. } \\
\text { Pain: Not measured. }\end{array}$ \\
\hline Mueller (2013) & $\begin{array}{l}\text { G1: Intervention- } \\
\text { Exercise WB. } \\
\text { G2: Intervention- } \\
\text { Exercise } \\
\text { NWB. }\end{array}$ & 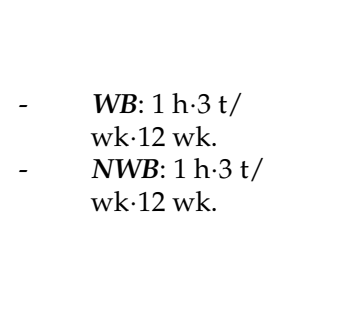 & $\begin{array}{l}\text { - WB: Group session. } \\
\text { Resistance exercise with } \\
\text { body weight + } \\
\text { treadmill. } \\
\text { NWB: Group session. } \\
\text { Exercise in sitting or } \\
\text { lying position with } \\
\text { elastic bands + cycle } \\
\text { ergometer. }\end{array}$ & $\begin{array}{ll}- & \text { 6MWT. } \\
- & \text { Step activity monitoring. } \\
- & \text { Foot and ankle ability measure. } \\
- & \text { Beck Depression Inventory-II. } \\
- & \text { 9-item physical performance test. } \\
- & \text { HbA1c. } \\
- & \text { Fat-free mass DAX. } \\
- & \text { PF peak torque. } \\
- & \text { ROM DF. }\end{array}$ & $\begin{array}{l}\text { G1 vs. G2: Significant differences } \\
(p<0.05) \text { in } 6 \mathrm{MWD} \text { for } \mathrm{G} 1 \text {, and in } \\
\text { HbA1c for } \mathrm{G} 2(p<0.05) .\end{array}$ & $\begin{array}{l}\text { Balance: Not measured. } \\
\text { Function: Better for G1 in } \\
\text { motor function. Better for } \\
\text { G2 in physiological } \\
\text { function. } \\
\text { Pain: Not measured. }\end{array}$ \\
\hline Nenkova (2009) & $\begin{array}{l}\text { G1: Intervention- } \\
\text { Exercise. } \\
\text { G2: Control. }\end{array}$ & $\begin{array}{l}\text { Exercise: } 6 \cdot 5 / 10 \\
\mathrm{~s} \cdot 45 \mathrm{~min} \cdot 3 \mathrm{t} / \mathrm{wk} \cdot 12 \\
\text { wk. } \\
\text { Control: } \mathrm{G} 1+\mathrm{G} 2.12 \\
\text { wk. }\end{array}$ & $\begin{array}{l}\text { Exercise: } 10 \mathrm{~min} \\
\text { warm-up }+25 \mathrm{~min} \\
\text { isometrics }+10 \mathrm{~min} \\
\text { cold-down. } \\
\text { Control: Daily } \\
\text { hypoglycaemic } \\
\text { medication + dietary } \\
\text { recommendations. }\end{array}$ & $\begin{array}{ll}- & \text { Romberg's test. } \\
\text { - } & \text { Force right and left quadriceps, } \\
\text { hamstrings, DF, PF. } \\
\text { Blood glucose level, blood } \\
\text { pressure, HR. }\end{array}$ & $\begin{array}{l}\text { G1 vs. G2: Significant differences } \\
(p<0.05) \text { in all outcomes for G1. }\end{array}$ & $\begin{array}{l}\text { Balance: Not measured. } \\
\text { Function: Better for G1. } \\
\text { Pain: Not measured. }\end{array}$ \\
\hline
\end{tabular}


Table 3. Cont

\begin{tabular}{|c|c|c|c|c|c|c|}
\hline Study & Groups & Dose & Description & Outcomes & Results & \\
\hline Quigley (2014) & $\begin{array}{l}\text { G1: Intervention- } \\
\text { Exercise FBT. } \\
\text { G2: Intervention- } \\
\text { Exercise TC. } \\
\text { G3: Control. }\end{array}$ & 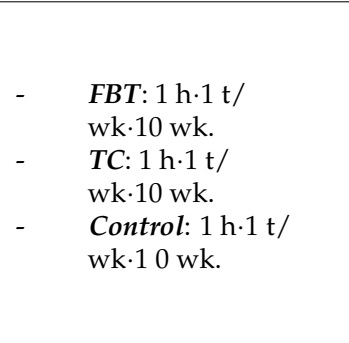 & $\begin{array}{ll}\text { - } & F B T: \text { Warm-up }+ \\
\text { increased-difficulty } \\
\text { program of functional } \\
\text { balance training. } \\
\text { TC: } 10 \text { min warm-up }+ \\
45 \text { min Tai-Chi }+5 \text { min } \\
\text { cold-down. } \\
\text { Control: Education. }\end{array}$ & $\begin{array}{ll}- & \text { BBS. } \\
- & \text { TUG. } \\
- & \text { Modified Falls Efficacy Scale. } \\
- & \text { Spatiotemporal gait variables. } \\
- & \text { Balance. }\end{array}$ & $\begin{array}{l}\text { G1: Significant improvement } \\
(p<0.05) \text { in peak ankle PF power, } \\
\text { peak ground reaction force-anterior } \\
\text { and posterior, step width and } \\
\text { variability. } \\
\text { G2: Significant improvement } \\
(p<0.05) \text { in TUG, step width and } \\
\text { step time. } \\
\text { G3: No significant improvement } \\
(p>0.05)\end{array}$ & $\begin{array}{l}\text { Balance: Improvement in } \\
\text { G1 and G2. } \\
\text { Function: Improvement } \\
\text { in G1 and G2. } \\
\text { Pain: Not measured. }\end{array}$ \\
\hline Richardson (2001) & $\begin{array}{l}\text { G1: Intervention- } \\
\text { Lower quadrant. } \\
\text { G2: Intervention- } \\
\text { Upper } \\
\text { quadrant. }\end{array}$ & $\begin{array}{l}\text { Lower quadrant: } \\
2 \cdot 10 \mathrm{rep} \cdot 1 \mathrm{t} / \mathrm{d} \cdot 3 \mathrm{wk} . \\
\text { Upper quadrant: } \\
>5 \mathrm{t} / \mathrm{wk} \cdot 3 \mathrm{wk} .\end{array}$ & $\begin{array}{l}\text { Lower quadrant: } \\
\text { Warm-up + } \\
\text { bipedal/unipedal toe } \\
\text { raises and heel raises + } \\
\text { bipedal/unipedal } \\
\text { inversion and eversion } \\
\text { + wall slides + unipedal } \\
\text { balance for time. } \\
\text { Upper quadrant: Upper } \\
\text { limb and cervical } \\
\text { exercise. }\end{array}$ & $\begin{array}{ll}\text { - } & \text { Tandem stance. } \\
\text { - } & \text { Functional reach. } \\
\text { - } & \text { Unipodal stance. } \\
\text { - } & \text { ABC scale. }\end{array}$ & $\begin{array}{l}\text { G1: Significant improvement } \\
(p<0.05) \text { in all outcomes except } \\
\text { ABC scale. } \\
\text { G2: No significant improvement } \\
(p>0.05) \text {. }\end{array}$ & $\begin{array}{l}\text { Balance: Improvement } \\
\text { in G1. } \\
\text { Function: Improvement } \\
\text { in G1. } \\
\text { Pain: Not measured. }\end{array}$ \\
\hline Serry (2016) & $\begin{array}{l}\text { G1: Intervention- } \\
\text { Exercise. } \\
\text { G2: Intervention- } \\
\text { TENS. } \\
\text { G3: Control. }\end{array}$ & $\begin{array}{l}\text { Exercise: } 50 \mathrm{~min} \cdot 3 \mathrm{t} / \\
\text { wk. } 8 \mathrm{wk} . \\
\text { TENS: } 30 \mathrm{~min} \cdot 3 \mathrm{t} / \\
\text { wk. } 8 \mathrm{wk} . \\
\text { - Control: } 8 \mathrm{wk} .\end{array}$ & $\begin{array}{ll}\text { - } & \text { Exercise: } 5 \mathrm{~min} \\
\text { warm-up }+40 \mathrm{~min} \\
\text { aerobic exercise }+5 \mathrm{~min} \\
\text { cold-down. } \\
\text { TENS: TENS } \\
\text { application. } \\
\text { Control: } \text { Medication. }\end{array}$ & $\begin{array}{ll}- & \text { VAS. } \\
- & \text { NCV. }\end{array}$ & $\begin{array}{l}\text { G1: Significant improvement } \\
(p<0.05) \text { in VAS. } \\
\text { G2: Significant improvement } \\
(p<0.05) \text { in VAS. } \\
\text { G3: No significant improvement } \\
(p>0.05) .\end{array}$ & $\begin{array}{l}\text { Balance: Not measured. } \\
\text { Function: No } \\
\text { improvement. } \\
\text { Pain: Improvement in G1 } \\
\text { and G2. }\end{array}$ \\
\hline $\begin{array}{l}\text { Seyedizadeh } \\
(2020)\end{array}$ & $\begin{array}{l}\text { G1: Intervention- } \\
\text { Exercise. } \\
\text { G2: Control. }\end{array}$ & $\begin{array}{ll}\text { - } & \text { Exercise: } 3 \mathrm{t} / \\
\text { - } & \text { Control: } 8 \mathrm{wk} . \\
\text { wk. }\end{array}$ & $\begin{array}{l}\text { Exercise: } 15 \text { min } \\
\text { warm-up }+8-12 \text { rep } \\
\text { resistance training }+ \\
3 \text { min interval aerobic } \\
\text { training }+15 \text { min } \\
\text { cold-down. } \\
\text { Control: } \text { Any } \\
\text { intervention. }\end{array}$ & $\begin{array}{ll}- & -6 \mathrm{MWT} . \\
- & -30 \text { s bicep curl test. } \\
- & \text { Rikli and Jones Chair Stand Test. } \\
\text { - } & \text { KLC1. }\end{array}$ & $\begin{array}{l}\text { G1: Significant improvement } \\
(p<0.05) \text { in lower limb strength. } \\
\text { G2: Significant improvement } \\
(p<0.05) \text { in lower limb strength. } \\
\text { G1 vs. G2: Significant differences } \\
(p<0.05) \text { in aerobic resistance and } \\
\text { lower limb strength for G1 }(p<0.05) .\end{array}$ & $\begin{array}{l}\text { Balance: No } \\
\text { improvement. } \\
\text { Function: Improvement } \\
\text { in G1 and G2. Better } \\
\text { for G1. } \\
\text { Pain: Not measured. }\end{array}$ \\
\hline
\end{tabular}


Table 3. Cont

\begin{tabular}{|c|c|c|c|c|c|c|}
\hline Study & Groups & Dose & Description & Outcomes & Results & \\
\hline Song (2011) & $\begin{array}{l}\text { G1: Intervention- } \\
\text { Exercise + } \\
\text { Education. } \\
\text { G2: Control. }\end{array}$ & $\begin{array}{l}\text { - } \quad \text { Exercise: } 2 \mathrm{t} / \\
\text { wk } 8 \mathrm{wk} / / \text { Education: } \\
50 \mathrm{~min} \cdot 1 \mathrm{t} / \mathrm{wk} \cdot 8 \mathrm{wk} \text {. } \\
\text { Control: } 8 \mathrm{wk} .\end{array}$ & $\begin{array}{l}\text { Exercise: } 10 \mathrm{~min} \\
\text { warm-up }+40 \mathrm{~min} \\
\text { balance train }+10 \mathrm{~min} \\
\text { cold-down. Health } \\
\text { education for diabetes. } \\
\text { Control: Any } \\
\text { intervention. }\end{array}$ & $\begin{array}{ll}\text { - } & \text { Postural sway path: EO AP, EO } \\
\text { ML, EO TS, EC AP, EC ML, EC TS. } \\
\text { - } & \text { OLS test: EO LT, EC LT, HRLT, EO } \\
& \text { RT, EC RT, HRRT. } \\
- & \text { BBS. } \\
- & \text { FRT. } \\
- & 10 \mathrm{~m} \text { walk. } \\
- & \text { EO, EC stable surface, EO foam. }\end{array}$ & $\begin{array}{l}\text { G1: Significant improvement } \\
(p<0.05) \text { for all outcomes. } \\
\text { G2: No significant improvement } \\
(p>0.05) .\end{array}$ & $\begin{array}{l}\text { Balance: Improvement } \\
\text { in G1. } \\
\text { Function: Improvement } \\
\text { in G1. } \\
\text { Pain: Not measured. }\end{array}$ \\
\hline Taveggia (2014) & $\begin{array}{l}\text { G1: Intervention- } \\
\text { Exercise. } \\
\text { G2: Control. }\end{array}$ & $\begin{array}{ll}\text { - } & \text { Exercise: } 20 \mathrm{~s} \cdot 5 \\
\text { t/wk } 4 \mathrm{wk} . \\
\text { - } \quad \text { Control: } 4 \mathrm{wk} .\end{array}$ & $\begin{array}{ll}\text { - } & \text { Exercise: } 20 \mathrm{~min} \\
\text { treadmill }+20 \mathrm{~min} \\
\text { isokinetic strength }+ \\
20 \text { min balance. } \\
\text { - } \quad \text { Control: } \text { Standard care. }\end{array}$ & $\begin{array}{ll}- & \text { 6MWT. } \\
- & 10 \mathrm{~m} \text { walking. } \\
- & \text { FIM. } \\
- & \text { Tinnetti scale walk. } \\
- & \text { SBP. } \\
- & \text { DBP. } \\
- & \text { RR. } \\
- & \text { SpO2. } \\
- & \text { VO2max. } \\
- & \text { REE. } \\
- & \text { VE. } \\
- & \text { FEO2. }\end{array}$ & $\begin{array}{l}\text { G1: Significant improvement } \\
(p<0.05) \text { in } 6 \text { MWT, FIM, SpO2. } \\
\text { G2: Significant improvement } \\
(p<0.05) \text { in 6MWT, FIM, SpO2. } \\
\text { G1 vs. G2: Significant differences in } \\
\text { Tinneti scale walk and FEO2 for G1 } \\
(p<0.05) .\end{array}$ & $\begin{array}{l}\text { Balance: Improvement in } \\
\text { G1 and G2. Better for G1. } \\
\text { Function: Improvement } \\
\text { in G1 and G2. Better for } \\
\text { G1. } \\
\text { Pain: Not measured. }\end{array}$ \\
\hline $\begin{array}{l}\text { Venkataraman } \\
\quad(2019)\end{array}$ & $\begin{array}{l}\text { G1: Intervention- } \\
\text { Exercise. } \\
\text { G2: Control. }\end{array}$ & $\begin{array}{l}\text { - } \quad \text { Exercise: } 1 \mathrm{t} / \\
\text { wk. } 8 \mathrm{wk} . \\
\text { - Control: } 8 \mathrm{wk} .\end{array}$ & $\begin{array}{l}\text { Exercise: } 5 \text { min } \\
\text { warm-up + } \\
\text { balance/strength }+ \\
5 \text { min cold-down. } \\
\text { Control: } \text { Routine care. }\end{array}$ & $\begin{array}{ll}- & \text { EQ-5D-5L. } \\
- & \text { SF-36v2. } \\
- & \text { TUG. } \\
- & \text { FTSTS. } \\
- & \text { ABC scale. }\end{array}$ & $\begin{array}{l}\text { G1 vs. G2: Significant differences in } \\
\text { HRQoL pain, TUG, FTSTS, ABC } \\
\text { scale, muscular strength (ankle and } \\
\text { knee) for G1 }(p<0.05) \text {. }\end{array}$ & $\begin{array}{l}\text { Balance: Better for G1. } \\
\text { Function: Better for G1. } \\
\text { Pain: Better for G1. }\end{array}$ \\
\hline
\end{tabular}


Table 3. Cont.

\begin{tabular}{|c|c|c|c|c|c|c|}
\hline Study & Groups & Dose & Description & Outcomes & Results & \\
\hline Win (2020) & $\begin{array}{l}\text { G1: Intervention- } \\
\text { Exercise. } \\
\text { G2: Control. }\end{array}$ & 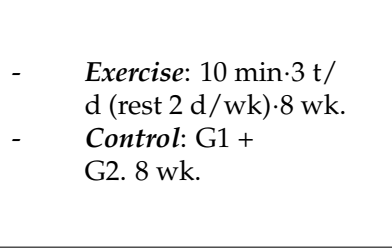 & $\begin{array}{l}\text { Exercise: Hand, toe and } \\
\text { foot exercise. } \\
\text { Control: Health } \\
\text { education. }\end{array}$ & $\begin{array}{ll}- & \text { PNQ. } \\
- & \text { BRS. } \\
- & \text { VAS. } \\
- & \text { SWM. } \\
- & \text { Hand grip. } \\
- & \text { Pinch force. } \\
- & \text { TUG. } \\
\end{array}$ & $\begin{array}{l}\text { G1 vs. G2: Significant differences in } \\
\text { PNQ (motor area) for G1 }(p<0.05) \text {. }\end{array}$ & $\begin{array}{l}\text { Balance: } \text { No significant } \\
\text { difference. } \\
\text { Function: Better for G1. } \\
\text { Pain: No significant } \\
\text { difference. }\end{array}$ \\
\hline \multicolumn{7}{|c|}{ b. Manual Therapy Intervention } \\
\hline Dalal (2014) & $\begin{array}{l}\text { G1: Intervention- } \\
\text { Reflexology. } \\
\text { G2: Control. }\end{array}$ & $\begin{array}{ll}\text { Reflexology: } \\
\quad 30 \mathrm{~min} \cdot 2 / \mathrm{d} \cdot 1 \mathrm{t} / \mathrm{m} \cdot 5 \mathrm{~m} \\
\text { Control: } \mathrm{G} 1+\mathrm{G} 25 \mathrm{~m}\end{array}$ & $\begin{array}{l}\text { Reflexology: } 15 \text { stimuli } \\
\text { for each foot area } \\
\text { during } 20 \mathrm{~s} . \\
\text { Control: Institute } \\
\text { standard mode of } \\
\text { pharmacological } \\
\text { management. }\end{array}$ & $\begin{array}{ll}- & \text { VAS. } \\
- & \text { NCV. } \\
- & \text { NeuroQoL test. } \\
- & \text { VPT. } \\
- & \text { CPT. } \\
- & \text { HPT. }\end{array}$ & $\begin{array}{l}\text { G1: Significant improvement } \\
(p<0.05) \text { in all outcomes. } \\
\text { G2: Significant improvement } \\
(p<0.05) \text { in all outcomes. } \\
\text { G1 vs. G2: Significant differences in } \\
\text { all outcomes for } \mathrm{G} 1(p<0.05) .\end{array}$ & $\begin{array}{l}\text { Balance: Not measured. } \\
\text { Function: Improvement } \\
\text { in both groups. Better for } \\
\text { G1. } \\
\text { Pain: Improvement in } \\
\text { both groups. Better for } \\
\text { G1. }\end{array}$ \\
\hline Gok Metin (2017) & $\begin{array}{l}\text { G1: Intervention- } \\
\text { Aromatherapy. } \\
\text { G2: Control }\end{array}$ & $\begin{array}{l}\text { Aromatherapy: } 30 \mathrm{~min} \cdot 12 \\
\text { wk. } 4 \mathrm{wk} \\
\text { Control: } 4 \mathrm{wk}\end{array}$ & $\begin{array}{l}\text { Aromatherapy: } 20 \mathrm{~min} \\
2 \mathrm{~s} \cdot 3 \mathrm{t} / \mathrm{massage} \text { feet }+10 \mathrm{~min} \\
\text { hands with essential oil. } \\
\text { - Control: Routine care }\end{array}$ & $\begin{array}{ll}- & \text { Patient questionnaire } \\
- & \text { DN4 } \\
- & \text { VAS } \\
- & \text { NePIQoL }\end{array}$ & $\begin{array}{l}\text { G1: Significant improvement } \\
(p<0.05) \text { for VAS, QoL. } \\
\text { G2: No significant improvement } \\
(p<0.05) \text {. } \\
\text { G1 vs. G2: Significant differences for } \\
\text { VAS and QoL for G1 }(p<0.05) \text {. }\end{array}$ & $\begin{array}{l}\text { Balance: Not measured. } \\
\text { Function: Improvement } \\
\text { in G1. Better for G1. } \\
\text { Pain: Improvement in G1. } \\
\text { Better for G1. }\end{array}$ \\
\hline Singh (2012) & $\begin{array}{l}\text { G1: Intervention- } \\
\text { Neurodynamic. } \\
\text { G2: Control. }\end{array}$ & $\begin{array}{l}\text { Neurodynamic: } \\
10 \mathrm{~min} / \mathrm{d} \cdot 21 \mathrm{~d} \\
-\quad \text { Control: } 21 \mathrm{~d}\end{array}$ & $\begin{array}{l}\text { Neurodynamic: } 30 \mathrm{~s} \\
\text { tibial nerve } \\
\text { mobilisation, } 1 \mathrm{~min} \\
\text { of rest. } \\
\text { Control: Any } \\
\text { intervention. }\end{array}$ & - $\quad$ VPT: 1st, 3rd, 5th metatarsal head. & $\begin{array}{l}\text { G1 vs. G2: Significant differences } \\
\text { for VPT 1st MTP right head for G1 } \\
(p<0.05)\end{array}$ & $\begin{array}{l}\text { Balance: Not measured. } \\
\text { Function: Better for G1. } \\
\text { Pain: Not measured. }\end{array}$ \\
\hline
\end{tabular}


Table 3. Cont.

\begin{tabular}{|c|c|c|c|c|c|c|}
\hline Study & Groups & Dose & Description & Outcomes & Results & \\
\hline Talebi (2018) & $\begin{array}{l}\text { G1: Intervention- } \\
\text { Manual therapy. } \\
\text { G2: Intervention- } \\
\text { TENS. }\end{array}$ & $\begin{array}{l}\text { Manual Therapy: } \\
25 \mathrm{~min} \cdot 3 \mathrm{t} / \mathrm{wk} \cdot 4 \mathrm{wk} . \\
\text { TENS: } 25 \mathrm{~min} \cdot 3 \mathrm{t} / \\
\text { wk. } 4 \mathrm{wk} .\end{array}$ & $\begin{array}{l}\text { Manual Therapy: } \\
\text { Carpal bone } \\
\text { mobilisation, transverse } \\
\text { carpal ligament release, } \\
\text { palmar fascia release of } \\
\text { the hand, soft tissue } \\
\text { manipulation in the } \\
\text { distal arm and proximal } \\
\text { forearm areas and } \\
\text { median nerve } \\
\text { mobilisation. } \\
\text { TENS: } 20 \text { min at } 80 \mathrm{~Hz} \\
\text { 60us pulse duration. } \\
\text { Comfortable tingling }+ \\
5 \text { min therapeutic US } \\
\text { on plantar heel. }\end{array}$ & $\begin{array}{ll}- & \text { VAS. } \\
- & \text { BCTQ (SSS-FSS). } \\
- & \text { MNT: angle of elbow. }\end{array}$ & $\begin{array}{l}\text { G1: Significant improvement } \\
(p<0.05) \text { in all outcomes. } \\
\text { G2: Significant improvement } \\
(p<0.05) \text { in VAS and BCQT-SSS. } \\
\text { G1 vs. G2: Significant differences in } \\
\text { SSS, FSS and MNT for G1 }(p<0.05) .\end{array}$ & $\begin{array}{l}\text { Balance: Not measured. } \\
\text { Function: Improvement } \\
\text { in both groups. Better } \\
\text { for G1. } \\
\text { Pain: Improvement in } \\
\text { both groups. }\end{array}$ \\
\hline Xie (2019) & $\begin{array}{l}\text { G1: Intervention- } \\
\text { Gua Sha therapy. } \\
\text { G2: Control. }\end{array}$ & $\begin{array}{l}\text { - Gua Sha therapy: } \\
60 \mathrm{~min} \cdot 12 \mathrm{~s} \cdot 1 \mathrm{t} / \\
\text { d. } 4 \mathrm{wk} . \\
\text { - Control: } 4 \mathrm{wk} .\end{array}$ & $\begin{array}{l}\text { Gua Sha therapy: Gua } \\
\text { Sha Oil massage along } \\
\text { midline of the back } \\
\text { with Gua Sha tool. } \\
\text { - Control: Standard care. }\end{array}$ & $\begin{array}{ll}- & \text { TCSS. } \\
- & \text { VPT. } \\
- & \text { ABI. } \\
- & \text { Fasting plasma glucose. }\end{array}$ & $\begin{array}{l}\text { G1: Significant improvement } \\
(p<0.05) \text { in all outcomes and } \\
\text { follow-ups. } \\
\text { G1 vs. G2: Significant differences in } \\
\text { VPT and ABI ( } 4 \mathrm{wk}) \text {; all outcomes } \\
(8 / 12 \mathrm{wk}) \text { for } \mathrm{G} 1(p<0.05) .\end{array}$ & $\begin{array}{l}\text { Balance: Not measured. } \\
\text { Function: Improvement } \\
\text { in G1. Better for G1. } \\
\text { Pain: Improvement in G1. }\end{array}$ \\
\hline
\end{tabular}


Table 3. Cont.

\begin{tabular}{|c|c|c|c|c|c|c|}
\hline Study & Groups & Dose & Description & Outcomes & Results & \\
\hline \multicolumn{7}{|c|}{ c. Combinate intervention } \\
\hline Shourabi (2020) & $\begin{array}{l}\text { G1: Intervention- } \\
\text { Aquatic exercise. } \\
\text { G2: Intervention- } \\
\text { AE + M. } \\
\text { G3: Intervention- } \\
\text { Massage. } \\
\text { G4: Control. }\end{array}$ & $\begin{array}{ll}\text { - } & \text { Aquatic exercise: } \\
& 60 \mathrm{~min} \cdot 3 \mathrm{t} / \mathrm{wk} \cdot 8 \mathrm{wk} . \\
\text { - } & \text { Massage: } 30 \mathrm{~min} \cdot 3 \mathrm{t} / \\
\text { wk } 8 \mathrm{wk} & \\
\text { Control: } 8 \mathrm{wk} .\end{array}$ & $\begin{array}{l}\text { Aquatic exercise: } \\
10 \text { min warm-up + } \\
40 \text { min walking, } \\
\text { balance and lower } \\
\text { extremity strength + } 10 \\
\text { cold-down. } \\
\text { Massage: Pressure } \\
\text { techniques, effleurage, } \\
\text { deep pressure, general } \\
\text { cycle of beats, pickup, } \\
\text { kneading with thumb, } \\
\text { pressing with the } \\
\text { forearm, compression } \\
\text { and caressing methods } \\
\text { for different body parts. } \\
\text { Control: Any } \\
\text { intervention. }\end{array}$ & $\begin{array}{ll}- & \text { Insulin. } \\
- & \text { HbA1c } \\
- & \text { Fasting blood sugar. } \\
- & \text { Nerve growth factor. } \\
- & \text { BBS. }\end{array}$ & $\begin{array}{l}\text { G1: Significant improvement } \\
(p<0.05) \text { in nerve growth factor and } \\
\text { BBS, and between G4 in favour of } \\
\text { G1. } \\
\text { G2: Significant improvement } \\
(p<0.05) \text { in all outcomes and } \\
\text { between G4 in favour of G2. } \\
\text { Signficant difference in BBS and } \\
\text { nerve growth factor between G1 and } \\
\text { G3, in favour of G2. } \\
\text { G3: Significant improvement } \\
(p<0.05) \text { in nerve growth factor and } \\
\text { BBS. }\end{array}$ & $\begin{array}{l}\text { Balance: Improvement in } \\
\text { G1, G2 and G3. Better for } \\
\text { G1 and G2 vs. G4, and G2 } \\
\text { vs. G1 and G3. } \\
\text { Function: Improvement } \\
\text { in G1, G2 and G3. Better } \\
\text { for G1 and G2 vs. G4, and } \\
\text { G2 vs. G1 and G3. } \\
\text { Pain: Not measured. }\end{array}$ \\
\hline
\end{tabular}

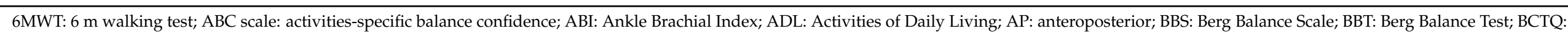

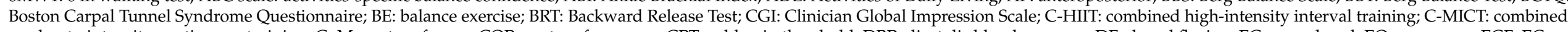

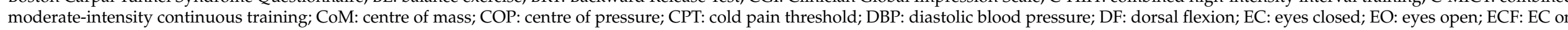

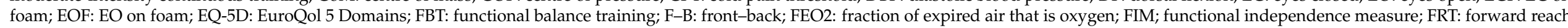

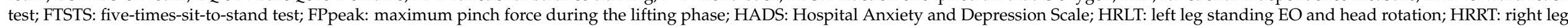

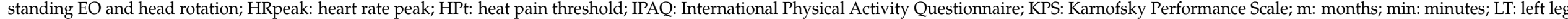

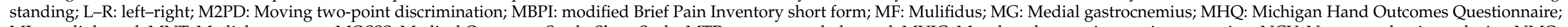

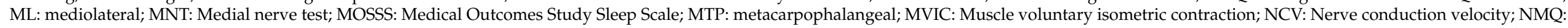

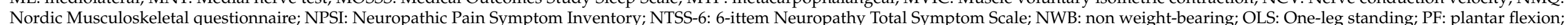

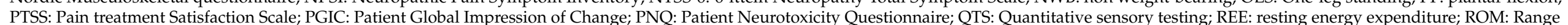

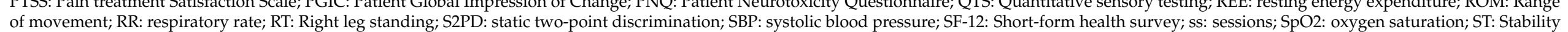

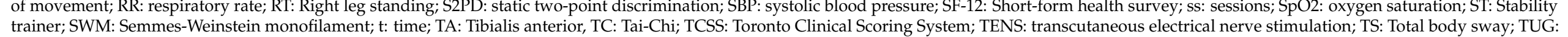

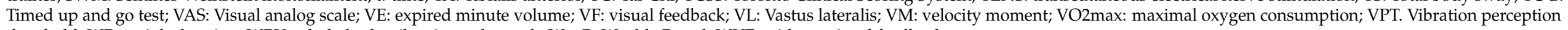
threshold; WB: weight-bearing; WBV: whole-body vibration; wk: week; WooB: Wooble Board; WVF: without visual feedback. 
The principal forms of exercise were balance work, aerobic exercise and strength exercises. There were 12 articles that developed various programmes to work on balance $[26,27,29-31,36-38,40,41,47,50]$. In this group, the studies included programmes focused on balance, sensory-motor exercises, gait exercises, bio-feedback and unstable platforms. Seven articles developed aerobic-type training [23,28,34,39,42,46,49]. These exercises were generally carried out at medium intensity using a treadmill or stationary bike. Lastly, four articles used strength exercises as the basis of their training [25,26,32,33]. The strength exercises were differentiated between using body weight or not, or strengthresistance training.

The variables used to analyse the results of this subgroup were mainly aimed at assessing balance. The assessments most used to analyse balance were as follows: postural sway was measured in seven articles $[28,29,40,41,47,49,50]$; the Berg Balance Test (BBT) was used in five articles $[30,37,39,47,49]$; the Timed Up and Go (TUG) test was used in five articles [26,27,30,37,47]; the Functional Reach Test (FRT), in four articles [36,37,39,49]; and the Five-Times-Sit-to-Stand (FTSTS) test, in two articles [26,37]. Other tests such as the $\mathrm{ABC}$ scale, the one-leg stance, the Tinnetti test and tandem test were also used to assess this outcome. Ten articles analysed function [23,25-27,29,31,32,38,46,50], using the Short Form12 Quality of Life (SF-12), the Neuropathy Total Symptom Score-6 (NTSS-6), health-related quality of life (EQ-5D) or the Functional Independence Measure (FIM) questionnaires, or tests such as quantitative sensory and range of movement tests. Pain was analysed in three studies using a visual analogue scale (VAS) $[27,34,46]$. Strength capacity was analysed in seven articles $[23,25,29,32,42,46,49]$ by means of the 6-Minute Walk Test (6MWT), the International Physical Activity Questionnaire (IPAQ), the maximum volume of oxygen consumption or the 10-Meter Walk Test.

The programmes focused on balance were compared with control groups with patient education and/or routine care, and statistically significant intra- and intergroup improvements were shown. In addition, the training that included vibration showed statistically significant differences as compared with a strength programme [38] or programmes without vibration [37]. Jannu et al. [30] added the wobble board against a balance programme, but they did not find statistically significant improvements in that group. This study used physiotherapy in both interventions. Training with a mini trampoline also observed statistically significant differences compared with a control group [50].

Aerobic training was also compared mainly with control groups; statistically significant differences in favour of the intervention group were found [28,42,46,49]. Cox et al. [23] observed that an exercise programme of high-intensity interval training (HIIT) and moderateintensity continuous training (MICT) showed improvement and significant differences in pain as compared with a control group. However, HIIT training might involve adverse effects if it exceeds $100 \mathrm{~h}$ of training. Turning to another focus, it seems that treadmill and stationary bike training is more effective than a balance training group [39]. Lastly, Serry et al. [34] observed that both aerobic exercise and electrotherapy improved pain significantly.

Essentially, strength training was compared with a control group. Mueller et al. [32], however, compared body-weight strength training with another strength training group without body-weight strength. They found statistically significant differences in the variable $\mathrm{HbA1c}$ in favour of the group with body weight, in the distance covered in the 6MWT test, and in favour of the group without body weight. The rest of the articles used various methodologies for strength training: isometrics [33], isokinetic exercises [25] and combined with balance [26]. All of these were effective in the balance and strength variables compared with the control group. Furthermore, the study that used isokinetic exercises (in addition) included a programme focused on stability and aerobic capacity. 


\section{Manual Therapy}

Only six articles included manual therapy as the intervention for patients with diabetic neuropathy. Once again, these studies compared manual therapy with control groups, which were defined as patient education, standard care or no intervention.

Thai-type massage [22], Gua Sha [43], reflexology [48] and aromatherapy [45] seem to be more effective in pain and function variables than the control group in patients with diabetic neuropathy. Treatment using neurodynamics yielded statistically significant favourable changes in the vibratory threshold in comparison with no intervention in these patients [24]. Lastly, the combination of articular, neural and soft tissue mobilisation techniques seemed to be more effective than an electrotherapy protocol in patients with diabetes having carpal tunnel syndrome [45].

In the case of manual therapy, the variables most used in the studies were pain (by a VAS scale) in three articles [44,45,48]; vibratory threshold in three articles [24,43,48]; and other variables linked to function such as questionnaires, sensitivity assessment or range of motion.

\section{Exercise and Manual Therapy}

Just one study [35] analysed the effect of an intervention with aquatic exercise, with massage and the combination of these two techniques against a control group (no intervention). Shourabi et al. observed statistically significant improvements in all the groups. In patients with diabetic neuropathy, the combination of aquatic exercise and massage was more effective for the variables related to balance and physiological factors.

\subsubsection{Dosage}

In the exercise group, the time used for the exercise programmes ranged from 40 to $60 \mathrm{~min}$. Most of the studies integrated warm-up and cool-down phases (from 5 to $10 \mathrm{~min}$ for each phase) in the intervention process. The total training period was some $60 \mathrm{~min}$ of intervention, principally in the aerobic and balance exercises. In contrast, the programmes focused on strength involved from 20 to 25 min of training.

As for hours a week, the majority of the protocols were given three times a week. The greatest number of sessions was once a day, in the study of Richardson et al. [36]. Dixit et al. [28] applied their intervention from 3 to 6 times a week. The minimum number of sessions per week (once a week) was stipulated in two articles [26,47].

Most of the articles used 8 weeks of follow-up to implement their study programmes [23,26-28,30,34,35,40-42,49,50]. Other studies had a follow-up of from 3 [36] up to 12 weeks. Toth et al. [46] used long-term follow-ups of up to 6 months, the maximum period in all the studies.

In the manual therapy group, the intervention period stipulated for all the studies was $30 \mathrm{~min}$. Only Singh et al. [24], with $10 \mathrm{~min}$ for the neurodynamic techniques, and Xie et al. [43], with $60 \mathrm{~min}$, differed from this period.

Treatment application varied among the articles. Treatment was given three times a week in three articles [22,44,45]; once a day in two studies [24,43]; and once a week in one article [48].

All of the studies carried out short-term follow-ups, from approximately 2 to 4 weeks. However, Dalal et al. [48] had a more long-term follow-up of 5 months.

The study of Shourabi et al. [35], which incorporated manual therapy and exercise groups into their study, also used the predominant doses in the rest of the studies in each speciality.

\subsubsection{Evaluation of Methodological Quality}

The RoB 2 tools show that the features with the worst methodological quality in the set of studies are the biases in measurement of the variable results, due to deviation from interventions, with approximately $25 \%$ being high risk. The biases from the lack of data on the variable results and from the selection of the results reported are the domains with 
the best methodological quality in the set of studies, around 75\% having a low risk of bias (Figure 2).

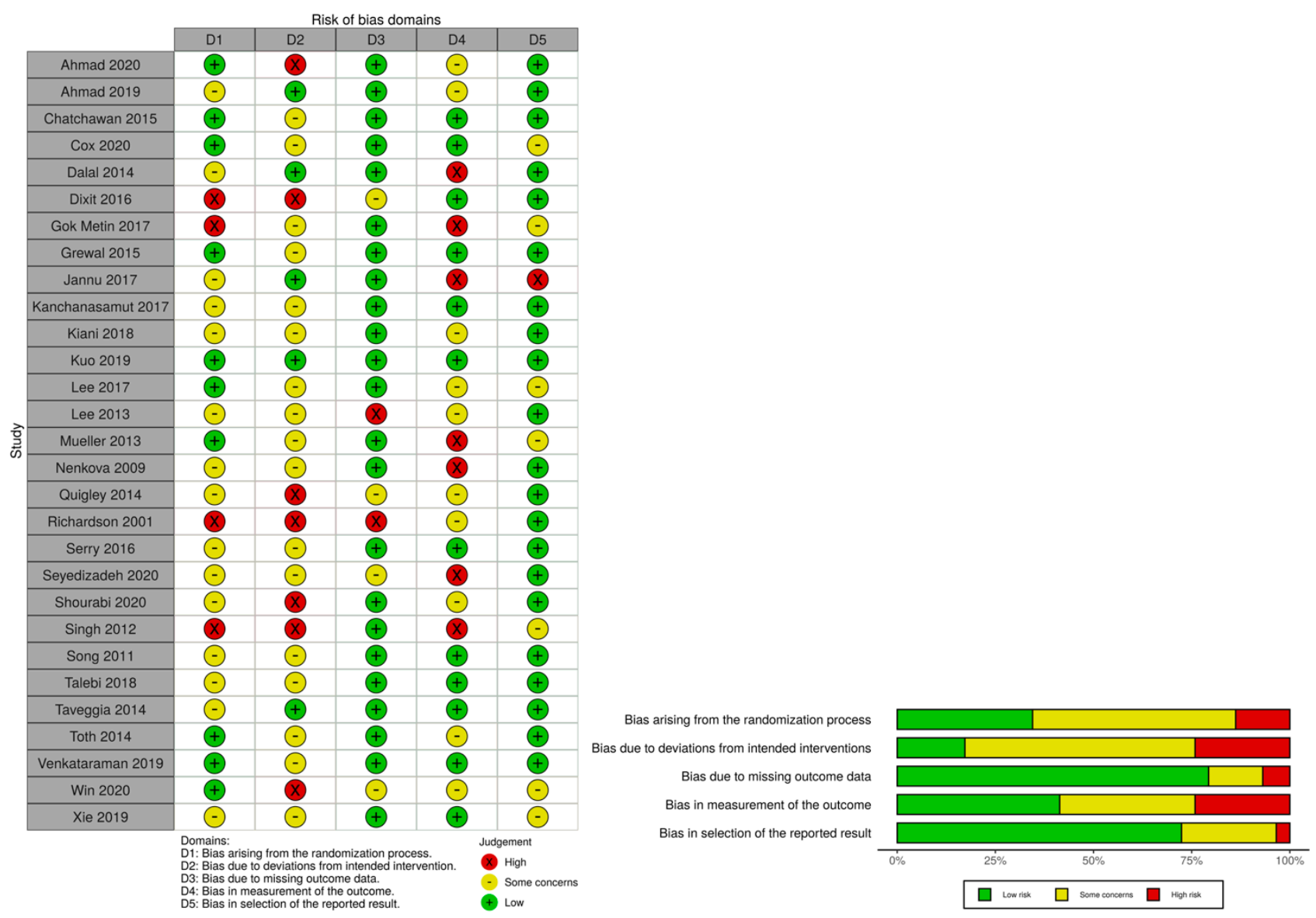

Figure 2. Risk of bias 2.0.

The quality measured in all the studies analysed using the PEDro scale was 6.6 out of 11 . Ten of the 29 studies analysed obtained an overall score of low quality $[24,27,30,33-36$, $39,40,50], 10$ articles received a moderate overall score [23,28,37,38,44,47-49] and 9 articles received a high overall score using this scale $[22,25,29,31,32,41-43,45,46]$ (Table 4). Some of the studies did not blind the subjects and the therapists that administered the therapy.

Table 4. PEDro Scale.

\begin{tabular}{|c|c|c|c|c|c|c|c|c|c|c|c|c|}
\hline Study & 1 & 2 & 3 & 4 & 5 & 6 & 7 & 8 & 9 & 10 & 11 & Total \\
\hline Ahmad (2020) & $x$ & $x$ & $X$ & $X$ & & & $x$ & $X$ & $X$ & $x$ & $x$ & 9 \\
\hline Ahmad (2019) & $X$ & $X$ & & $X$ & & & & & & $x$ & $X$ & 5 \\
\hline Chatchawan (2015) & $X$ & $X$ & $X$ & $X$ & & & $x$ & $X$ & $X$ & $X$ & & 8 \\
\hline Cox $(2020)$ & $x$ & $x$ & $x$ & $x$ & & & & & $x$ & $x$ & $X$ & 7 \\
\hline Dalal (2014) & $x$ & $x$ & & $x$ & & & & $x$ & & $x$ & $x$ & 6 \\
\hline Dixit (2016) & $x$ & $x$ & & $x$ & & & $X$ & & & $x$ & $X$ & 6 \\
\hline Gok Metin (2017) & $X$ & $x$ & & $x$ & & & $x$ & $x$ & $x$ & $X$ & $x$ & 8 \\
\hline Grewal (2015) & $X$ & $X$ & $X$ & $X$ & & & & $x$ & $X$ & $x$ & $X$ & 8 \\
\hline Jannu (2017) & & $x$ & & $X$ & & & & & & $x$ & $X$ & 5 \\
\hline Kanchanasamut (2017) & $X$ & & & $X$ & & & & & & $x$ & $X$ & 4 \\
\hline Kiani (2018) & $x$ & $x$ & & $x$ & & & & & & $x$ & $x$ & 5 \\
\hline Kuo (2019) & $X$ & $X$ & $X$ & $X$ & & & $X$ & $x$ & $X$ & $X$ & $X$ & 9 \\
\hline Lee (2017) & $x$ & $x$ & & $X$ & & & $x$ & $x$ & & $x$ & $X$ & 7 \\
\hline Lee (2013) & $x$ & $x$ & & $X$ & & & $X$ & $x$ & & $x$ & $X$ & 7 \\
\hline
\end{tabular}


Table 4. Cont

\begin{tabular}{|c|c|c|c|c|c|c|c|c|c|c|c|c|}
\hline Study & 1 & 2 & 3 & 4 & 5 & 6 & 7 & 8 & 9 & 10 & 11 & Total \\
\hline Mueller (2013) & $x$ & $x$ & $x$ & $x$ & & & $x$ & $x$ & $x$ & $x$ & $x$ & 9 \\
\hline Nenkova (2009) & $x$ & & & $x$ & & & & & & $x$ & $x$ & 4 \\
\hline Quigley (2014) & $x$ & $X$ & $X$ & $X$ & & & $X$ & & & $X$ & $x$ & 7 \\
\hline Richardson (2001) & $x$ & & & $x$ & & & & & & $x$ & $x$ & 4 \\
\hline Serry (2016) & $X$ & $x$ & & $x$ & & & & & & $x$ & $x$ & 5 \\
\hline Seyedizadeh (2020) & $X$ & $X$ & & $X$ & & & $X$ & $X$ & $X$ & $X$ & $X$ & 8 \\
\hline Shourabi (2020) & $x$ & $x$ & & $x$ & & & & $x$ & & $x$ & & 5 \\
\hline Singh (2012) & $x$ & $x$ & & & & & & & & $x$ & $x$ & 4 \\
\hline Song (2011) & $X$ & $x$ & & $x$ & & & & $x$ & & $X$ & $x$ & 6 \\
\hline Talebi (2018) & $X$ & $X$ & & $X$ & & & $X$ & $x$ & & $X$ & $x$ & 7 \\
\hline Taveggia (2014) & $x$ & $x$ & & $x$ & & & $x$ & $x$ & & $x$ & $x$ & 8 \\
\hline Toth (2014) & $x$ & $x$ & $x$ & $x$ & & & $x$ & $x$ & $X$ & $x$ & $x$ & 9 \\
\hline Venkataraman (2019) & $x$ & $x$ & & $x$ & & & & & & $x$ & $x$ & 7 \\
\hline Win $(2020)$ & $x$ & $X$ & & $X$ & & & & & & $X$ & $x$ & 5 \\
\hline Xie (2019) & $x$ & $x$ & $x$ & $x$ & & & $x$ & $x$ & & $x$ & $x$ & 8 \\
\hline Mean & & & & & & & 6.6 & & & & & \\
\hline
\end{tabular}

\section{Discussion}

The objective of this systematic review was to review the effectiveness of manual therapy and exercise in patients with diabetic neuropathy. We found 29 articles, of which just one analysed the combination of both techniques. There were six articles that analysed the effect of manual therapy, while the rest analysed a variety of exercise programmes. We found a lack of studies that analysed the combination of manual therapy and exercise and only manual therapy in patients with diabetic neuropathy.

The selection criteria were heterogeneous among the studies. Beginning with sample age, the articles differed in the minimum age for sample inclusion, from 18 to 40 or 45 years. The mean age ranges were between 45 and 85 years old. In other reviews, the mean ages of the patients were between 50 and 60 years old [10,12]. This can be explained by the elevated diagnosis of diabetic neuropathy in the 51-60-year age group, in patients that have type 2 diabetes [51,52]. In addition, type 2 diabetes represents the greatest proportion of the total prevalence of diabetes (90\%) and is characteristic of adult patients [53].

In line with the previous point, a great number of the analysed studies specified that the patients had to have type 2 diabetes, excluding patients with type 1 from the sample. The studies that include these patients do not provide results based on the type of diabetes that the patients have. Consequently, it is impossible to determine the effectiveness of these treatments in those patients and on diabetic neuropathy.

Moreover, the typology of diabetic neuropathy was defined as diabetic peripheral neuropathy, not considering other types of diabetic neuropathy such as diabetic autonomic neuropathy, cardiac autonomic neuropathy, gastrointestinal neuropathy and genitourinary disturbances. These profiles should also be well identified in the selection criteria because of the problems that exercise can have on patients [14].

Another point to be mentioned is the heterogeneity of the criteria used to diagnose diabetic neuropathy. The articles describe the use of the Michigan Diabetic Neuropathy (MDN) questionnaire, medical diagnoses, sensitivity tests and blood parameters (HbA1c and blood glycaemia). Nagpal et al. [54] observed that the predictive models for diagnosing diabetic neuropathy were ambiguous; electrodiagnostic studies do not enable detecting lesions in fibres of small diameter (which are the most damaged in this type of patient); imaging tests present different limitations and their usefulness has not yet been demonstrated in this type of clinical picture; and biomarkers need further studies [55]. Consequently, no agreement has been reached as to which criterion or criteria should be taken into account in diagnosing diabetic neuropathy. The studies should unify criteria so as to obtain a more representative sample that consider the alterations at the level of both large- and small-diameter nerve fibres. 
We have found considerable variability in the treatment with exercise. This variability exists in both the method of exercise and in the analysed variables. All the forms of treatment (aerobic exercise, balance therapy and strength-resistance treatment, as well as the combination among them) seem to be effective in improving the signs and symptoms of patients with diabetic neuropathy. Aerobic exercise, described principally as walking or using a stationary bike at moderate intensity, significantly improved all the variables analysed in the studies. Statistically significant differences were shown against control groups of standard care and/or patient education, but these results were not observed with groups using TENS interventions. Kiani et al. [39] showed improvements using several questionnaires in relation to a group treated with balance-focused exercise. In their systematic review, Gu et al. [56] also found that moderate-intensity aerobic exercise positively impacted neural function in patients having type 2 diabetes with diabetic neuropathy.

Balance programmes were also developed in a very heterogeneous way. The programmes using vibration, biofeedback, Tai-Chi and sensory-motor techniques seemed to have significant improvements after the exercise programme and to have the best benefit compared with control groups of standard care and/or education. Jannu et al. [30] observed that using the wobble board did not yield any benefits against a stability programme. A biofeedback protocol [31] demonstrated significant improvements compared with a multimodal treatment using tendon gliding, resistance training and patient care. The programmes that used vibration also had statistically significant improvements in functional variables as compared with standard strength or balance exercises. In their systematic review, Ites et al. [57] found that the intervention using exercise centred on the lower limb was recommendable, for clinical use, in treating balance dysfunction in diabetic neuropathy. However, they stressed that there were few high-quality studies on this research area.

In our review, we found that the studies using this type of programme were of moderate to high quality. Using these programmes makes it possible to conserve motor control and balance (in spite of sensory deficit) and prevent falls or hyperpressure areas, avoiding ulcerous processes and amputations of the feet. However, just a single study [30] included conventional physiotherapy as a complement to balance training, evading the effects that flexibility or postural re-education programmes can implement to structurally balance the supports, not only at the sensitive level.

The analysed strengthening programmes also yielded benefits in the signs and symptoms of patients with diabetic neuropathy. Although the evidence in this sense is more scarce, this type of exercise is beneficial (whether in isolation or in combination with the two previous types of exercise) in balance and function variables. The American Diabetes Association [14] encourages including strength training for these patients to mitigate neural symptoms and conserve muscle mass in the elderly, as well as to improve quality of life and hyperglycaemia control.

Exercise, in all its modalities, is beneficial for patients with diabetic neuropathy, with high methodological quality of the studies included. However, the variety of the modes used makes it impossible to identify the most beneficial type of exercise for patients with diabetic neuropathy. The American Diabetes Association [14] recommends doing a minimum of $150 \mathrm{~min} /$ week of moderate-intensity aerobic exercise or getting $75 \mathrm{~min} /$ week of vigorous aerobics, at a minimum of three times a week with a rest between sessions of no more than two consecutive days; $2-3$ sessions/week of non-consecutive resistance exercises; and flexibility and balance training 2-3 times/week. Of the studies included in this systematic review, only five $[22,27,33,39,40]$ reached these recommendations. Furthermore, the variety of doses used and of follow-ups make it impossible to specify which type of dosage is the most beneficial for the patients. It would be interesting to design studies based on the recommendations established by the ADA, as well as monitoring cardiovascular parameters (altered in this collective) [14].

As for manual therapy, there seems to be a short-term effect of the different types of massage after treatment in comparison with standard-care and treatment-free control 
groups, with high methodological quality of the studies. In the case of neurodynamics, improvements are also observed after treatment at the vibratory threshold. Lastly, Talebi et al. [44] observed that an intervention involving articular, neural and soft tissue presented greater benefits than using TENS in patients with diabetes and carpal tunnel syndrome, with moderate methodological quality. It should be pointed out that the only study with medium-term follow-up (5 months) that observed benefits as compared with conventional drug treatment was the study that was of the highest methodological quality. As Zhu et al. [58] observed in a study on rats with diabetic neuropathy, using neural techniques acted on the symptoms and reduced pro-inflammatory cytokines. In addition, it was observed that patients with type 1 diabetes present increased synthesis of type III collagen, which reflects the deposit of matrix and connective tissue in the basal membrane [59]. Manual therapy by local intervention may help to control the symptoms. It has been shown to act at the peripheral, spinal and supra-spinal levels [60]. The mechanical stimuli that such treatment provides favours the microcirculation in tissues, even in nerves. Approaching all the surrounding tissues, such as the joint, soft tissue and even the nerve itself, is more effective against the symptoms because of the increased oxygenation of all the tissues [61].

Shourabi et al. [35] were the only ones to study the combination of the two modalities. It was seen that the combination of both was more beneficial than aquatic exercise and massage alone. However, these results cannot be compared with those of the rest of the studies due to the different variables evaluated and the moderate quality of the study. Lastly, due to the limited number of studies that compared both modalities and the diversity of result variables used to quantify the effects of the techniques, it is impossible for us to conclude whether the combination of manual therapy and exercise is the most effective for the treatment of patients with diabetic neuropathy.

The results of this systematic review have some caveats due to the limitations of the studies included. The lack of homogeneity in the sample selection criteria, along with the variety of result variables used, make it difficult to compare the studies. Special mention should be made of the lack of specific reference in the studies to the procedures carried out for the detection in patients of the possible presence of cardiovascular autonomic neuropathy. Only two studies $[39,41]$ established it as an exclusion criterion, and did not specify the diagnostic criteria used for it. Neither did they include the presence of ischemic heart disease in the exclusion criteria. Examination for cardiovascular autonomic neuropathy is essential before prescribing exercise because it can be asymptomatic and detected only by the lack of variability of the heart rate with respiration, and it is associated with an increased cardiovascular risk [14].

The diversity of exercise modalities and manual techniques also makes objective comparison of the results difficult. The review itself is subject to limitations stemming from the wide range of result variables included for analysis.

\section{Conclusions}

In short, the various modes of exercise and manual therapy are beneficial for patients with diabetic peripheral neuropathy. Regarding the combination of both therapies, no conclusion on this hypothesis can be drawn due to the lack of evidence available. Exercise has been widely studied in its different modalities, but new revisions by modes of exercise, with similar result variables, should be carried out to establish more specific protocols. As for manual therapy, the limited number of studies on these patients must be increased in order to obtain greater knowledge about its effect. Finally, in the face of the benefits from both techniques separately, increasing the amount of evidence on the effect of combining them is needed for verifying its potential effectiveness for these patients.

Author Contributions: Conceptualisation, M.H.-S., M.O.L.-L., J.M.T.-M. and C.H.-G.; methodology, M.H.-S.; investigation, M.H.-S., M.O.L.-L. and C.H.-G.; writing-original draft preparation, M.H.-S., M.O.L.-L., C.V.-P., and C.H.-G.; writing-review and editing, S.M.-G., J.C.-d.-T., J.M.-T.-U. and J.R.-S. All authors have read and agreed to the published version of the manuscript. 
Funding: This research received no external funding.

Institutional Review Board Statement: Not applicable.

Informed Consent Statement: Not applicable.

Conflicts of Interest: The authors declare no conflict of interest.

\section{References}

1. American Diabetes Association. Diagnosis and Classification of Diabetes Mellitus. Diabetes Care 2005, 28 , 7-42.

2. Wild, S.; Roglic, G.; Green, A.; Sicree, R.; King, H. Global prevalence of diabetes: Estimates for the year 2000 and projections for 2030. Diabetes Care 2004, 27, 1047-1053. [CrossRef]

3. Van Laake-Geelen, C.C.M.; Smeets, R.J.E.M.; Quadflieg, S.P.A.B.; Kleijnen, J.; Verbunt, J.A. The effect of exercise therapy combined with psychological therapy on physical activity and quality of life in patients with painful diabetic neuropathy: A systematic review. Scand. J. Pain 2019, 19, 433-439. [CrossRef] [PubMed]

4. Tu, Y.; Lineaweaver, W.C.; Chen, Z.; Hu, J.; Mullins, F.; Zhang, F. Surgical Decompression in the Treatment of Diabetic Peripheral Neuropathy: A Systematic Review and Meta-analysis. J. Reconstr. Microsurg. 2016, 33, 151-157. [CrossRef]

5. Tadesse, D.B.; Gebrewahd, G.T.; Hailay, A.; Aberhe, W.; Mebrahtom, G.; Zereabruk, K.; Gebreayezgi, G.; Mariye, T.; Haile, T.G.; Gebremeskel, G.G.; et al. Diabetic Peripheral Neuropathy in Ethiopia: A Systematic Review and Meta-Analysis. J. Diabetes Res. 2021, 2021, 5304124.

6. Castelli, G.; Desai, K.M.; Cantone, R.E. Peripheral Neuropathy: Evaluation and Differential Diagnosis. Am. Fam. Physician 2020, 102, 732-739.

7. Vincent, A.M.; Brownlee, M.; Russell, J.W. Oxidative stress and programmed cell death in diabetic neuropathy. N. Y. Acad. Sci. 2002, 959, 368-383. [CrossRef] [PubMed]

8. Samper Bernal, D.; Monerris Tabasco, M.M.; Homs Riera, M.; Soler Pedrola, M. Aetiology and management of diabetic peripheral neuropathy. Rev. Soc. Española Dolor. 2010, 17, 286-296. [CrossRef]

9. Vázquez San Miguel, F.; Mauricio Puente, D.; Viadé Julià, J. Neuropatía diabética y pie diabético. Medicine 2016, 12, 971-981. [CrossRef]

10. Wong, M.C.; Chung, J.W.Y.; Wong, T.K.S. Effects of treatments for symptoms of painful diabetic neuropathy: Systematic review. Br. Med. J. 2007, 335, 87-90. [CrossRef]

11. Stein, C.; Eibel, B.; Sbruzzi, G.; Lago, P.D.; Plentz, R.D.M. Electrical stimulation and electromagnetic field use in patients with diabetic neuropathy: Systematic review and meta-analysis. Braz. J. Phys. Ther. 2013, 17, 93-104. [CrossRef] [PubMed]

12. Yammine, K.; Wehbe, R.; Assi, C. A systematic review on the efficacy of vitamin D supplementation on diabetic peripheral neuropathy. Clin. Nutr. 2020, 39, 2970-2974. [CrossRef]

13. Colberg, S.R.; Sigal, R.J.; Fernhall, B.; Regensteiner, J.G.; Blissmer, B.J.; Rubin, R.R.; Chasan-Taber, L.; Albright, A.L.; Braun, B. Exercise and type 2 diabetes: The American College of Sports Medicine and the American Diabetes Association: Joint position statement. Diabetes Care 2010, 33, 147-167. [CrossRef] [PubMed]

14. Association, A.D. Standards of Diabetes Care-2021. J. Clin. Appl. Res. Educ. 2021, 44, S21-S226.

15. Matthews, B.G.; Hurn, S.E.; Harding, M.P.; Henry, R.A.; Ware, R.S. The effectiveness of non-surgical interventions for common plantar digital compressive neuropathy (Morton's neuroma): A systematic review and meta-analysis. J. Foot Ankle Res. 2019, 12, 1-21. [CrossRef]

16. Del Barrio, S.J.; Gracia, E.B.; García, C.H.; de Miguel, E.E.; Moreno, J.T.; Marco, S.R.; Laita, L.C. Tratamiento conservador en pacientes con síndrome del túnel carpiano con intensidad leve o moderada. Revisión sistemática. Neurología 2018 , 33, 590-601. [CrossRef]

17. Hernández-Secorún, M.; Montaña-Cortés, R.; Hidalgo-García, C.; Rodríguez-Sanz, J.; Corral-de-Toro, J.; Monti-Ballano, S.; Hamam-Alcober, S.; Tricás-Moreno, J.M.; Lucha-López, M.O. Effectiveness of conservative treatment according to severity and systemic disease in carpal tunnel syndrome: A systematic review. Int. J. Environ. Res. Public Health 2021, 18, 2365. [CrossRef] [PubMed]

18. Page, M.J.; McKenzie, J.E.; Bossuyt, P.M.; Boutron, I.; Hoffmann, T.C.; Mulrow, C.D.; Shamseer, L.; Tetzlaff, J.M.; Moher, D. The PRISMA 2020 statement: An updated guideline for reporting systematic reviews. BMJ 2021, 372, n71. [CrossRef]

19. Herbert, R.; Moseley, A.; Sherrington, C.; Maher, C. Escala PEDro-Español. Physiotherapy 2000, 86, 55. [CrossRef]

20. Sterne, J.A.; Savović, J.; Page, M.J.; Elbers, R.G.; Blencowe, N.S.; Boutron, I.; Cates, C.J.; Cheng, H.Y.; Corbett, M.S.; Eldridge, S.M.; et al. RoB 2: A revised tool for assessing risk of bias in randomised trials. BMJ 2019, 366, 14898. [CrossRef]

21. Risk of Bias Tools-Robvis (Visualization Tool). Cochrane Database of Systematic Reviews. Available online: https://mcguinlu. shinyapps.io/robvis/ (accessed on 10 March 2021).

22. Chatchawan, U.; Eungpinichpong, W.; Plandee, P.; Yamauchi, J. Effects of thai foot massage on balance performance in diabetic patients with peripheral neuropathy: A randomized parallel-controlled trial. Med. Sci. Monit. Basic Res. 2015, $21,68-75$.

23. Cox, E.R.E.; Gajanand, T.; Burton, N.W.N.; Coombes, J.J.S.; Coombes, B.B.K. Effect of different exercise training intensities on musculoskeletal and neuropathic pain in inactive individuals with type 2 diabetes-Preliminary randomised controlled trial [with consumer summary]. Diabetes Res. Clin. Pract. 2020, 164, 108168. [CrossRef] 
24. Singh, P.P.; Bindra, S.; Singh, S.; Aggarwal, R.; Singh, J. Effect of Nerve Mobilization on Vibration Perception Threshold in Diabetic Peripheral Neuropathy. Indian J. Physiother. Occup. Ther. 2012, 6, 189-195.

25. Taveggia, G.; Villafañe, J.H.; Vavassori, F.; Lecchi, C.; Borboni, A.; Negrini, S. Multimodal treatment of distal sensorimotor polyneuropathy in diabetic patients: A randomized clinical trial. J. Manip. Physiol. Ther. 2014, 37, 242-252. [CrossRef]

26. Venkataraman, K.; Tai, B.C.; Khoo, E.Y.; Tavintharan, S.; Chandran, K.; Hwang, S.W.; Phua, M.S.; Wee, H.L.; Koh, G.C.; Tai, E.S. Short-term strength and balance training does not improve quality of life but improves functional status in individuals with diabetic peripheral neuropathy: A randomised controlled trial. Diabetologia 2019, 62, 2200-2210. [CrossRef]

27. Win, M.M.T.M.M.; Fukai, K.; Nyunt, H.H.H.; Linn, K.Z.K. Hand and foot exercises for diabetic peripheral neuropathy: A randomized controlled trial. Nurs. Health Sci. 2020, 22, 416-426. [CrossRef] [PubMed]

28. Dixit, S.; Maiya, A.; Shastry, B.A.; Guddattu, V. Analysis of postural control during quiet standing in a population with diabetic peripheral neuropathy undergoing moderate intensity aerobic exercise training: A single blind, randomized controlled trial. Am. J. Phys. Med. Rehabil. 2016, 95, 516-524. [CrossRef]

29. Grewal, G.S.; Schwenk, M.; Lee-Eng, J.; Parvaneh, S.; Bharara, M.; Menzies, R.A.; Talal, T.K.; Armstrong, D.G.; Najafi, B Sensor-Based Interactive Balance Training with Visual Joint Movement Feedback for Improving Postural Stability in Diabetics with Peripheral Neuropathy: A Randomized Controlled Trial. Gerontology 2015, 61, 567-574. [CrossRef] [PubMed]

30. Jannu, C.; Prathap, S.; Puchchakayala, G.; Vahini, C.; Balaraju, K. Efficacy of Stability Trainer Exercises versus Wobble Board Exercises in Balance Re-Education in Patients with Diabetic Neuropathy. Indian J. Physiother. Occup. Ther. An. Int. J. 2017, 11, 12. [CrossRef]

31. Kuo, L.C.; Yang, C.J.; Lin, C.F.; Jou, I.M.; Yang, Y.C.; Yeh, C.H.; Lin, C.C.; Hsu, H.Y. Effects of a task-based biofeedback training program on improving sensorimotor function in neuropathic hands in diabetic patients: A randomized controlled trial. Eur. J. Phys. Rehabil. Med. 2019, 55, 618-626. [CrossRef] [PubMed]

32. Mueller, M.J.; Tuttle, L.J.; LeMaster, J.W.; Strube, M.J.; McGill, J.B.; Hastings, M.K.; Sinacore, D.R. Weight-bearing versus nonweight-bearing exercise for persons with diabetes and peripheral neuropathy: A randomized controlled trial. Arch. Phys. Med. Rehabil. 2013, 94, 829-838. [CrossRef]

33. Nenkova, S.; Stewart, A.; Potterton, J.; Becker, P. The effects of isometric exercises and stretching on postural stability in NonInsulin Dependent Diabetes Mellitus patients with diffuse symmetrical sensory motor neuropathy. S. Afr. J. Physiother. 2009, 65, 27-31. [CrossRef]

34. Serry, Z.M.; Mossa, G.; Elhabashy, H.; Elsayed, S.; Elhadidy, R.; Azmy, R.M.; Mokhtar, A. Transcutaneous nerve stimulation versus aerobic exercise in diabetic neuropathy. Egypt J. Neurol. Psychiatry Neurosurg. 2016, 53, 124-129.

35. Shourabi, P.; Bagheri, R.; Ashtary-Larky, D.; Wong, A.; Motevalli, M.S.; Hedayati, A.; Baker, J.S.; Rashidlamir, A. Effects of hydrotherapy with massage on serum nerve growth factor concentrations and balance in middle aged diabetic neuropathy patients. Complement. Ther. Clin. Pract. 2020, 39, 101141. [CrossRef] [PubMed]

36. Richardson, J.K.; Sandman, D.; Vela, S. A focused exercise regimen improves clinical measures of balance in patients with peripheral neuropathy. Arch. Phys. Med. Rehabil. 2001, 82, 205-209. [CrossRef] [PubMed]

37. Lee, K.; Lee, S.; Song, C. Whole-body vibration training improves balance, muscle strength and glycosylated hemoglobin in elderly patients with diabetic neuropathy. Tohoku J. Exp. Med. 2013, 231, 305-314. [CrossRef]

38. Lee, K. Effects of whole-body vibration therapy on perception thresholds of type 2 diabetic patients with peripheral neuropathy: A randomized controlled trial. J. Phys. Ther. Sci. 2017, 29, 1684-1688. [CrossRef]

39. Kiani, N.; Marryam, M.; Malik, A.A.N.; Amjad, I. The effect of aerobic exercises on balance in diabetic neuropathy patients. J. Med. Sci. 2018, 26, 141-145.

40. Ahmad, I.; Noohu, M.M.; Verma, S.; Singla, D.; Hussain, M.E. Effect of sensorimotor training on balance measures and proprioception among middle and older age adults with diabetic peripheral neuropathy. Gait Posture 2019, 74, 114-120. [CrossRef]

41. Ahmad, I.; Verma, S.; Noohu, M.M.; Shareef, M.Y.; Ejaz Hussain, M. Sensorimotor and gait training improves proprioception, nerve function, and muscular activation in patients with diabetic peripheral neuropathy: A randomized control trial. J. Musculoskelet Neuronal Interact. 2020, 20, 234-248.

42. Seyedizadeh, S.S.H.; Cheragh-Birjandi, S.; Hamedi Nia, M.R.M.; Cheragh-Birjandi, S.; Hamedi Nia, M.R.M. The Effects of Combined Exercise Training (Resistance-Aerobic) on Serum Kinesin and Physical Function in Type 2 Diabetes Patients with Diabetic Peripheral Neuropathy (Randomized Controlled Trials). J. Diabetes Res. 2020, 2020, 6978128. [CrossRef] [PubMed]

43. Xie, X.; Lu, L.; Zhou, X.; Zhong, C.; Ge, G.; Huang, H.; Zhang, X.; Zeng, Y. Effect of Gua Sha therapy on patients with diabetic peripheral neuropathy: A randomized controlled trial. Complement. Ther. Clin. Pract. 2019, 35, 348-352. [CrossRef]

44. Talebi, G.A.; Saadat, P.; Javadian, Y.; Taghipour, M. Manual therapy in the treatment of carpal tunnel syndrome in diabetic patients: A randomized clinical trial. Casp. J. Intern. Med. 2018, 9, 283-289.

45. Gok Metin, Z.; Arikan Donmez, A.; Izgu, N.; Ozdemir, L.; Arslan, I.E. Aromatherapy Massage for Neuropathic Pain and Quality of Life in Diabetic Patients. J. Nurs. Scholarsh. 2017, 49, 379-388. [CrossRef]

46. Toth, C.; Brady, S.; Gagnon, F.; Wigglesworth, K. A randomized, single-blind, controlled, parallel assignment study of exercise versus education as adjuvant in the treatment of peripheral neuropathic pain. Clin. J. Pain 2014, 30, 111-118. [CrossRef]

47. Quigley, P.A.; Bulat, T.; Schulz, B.; Friedman, Y.; Hart-Hughes, S.; Richardson, J.K.; Barnett, S. Exercise interventions, gait, and balance in older subjects with distal symmetric polyneuropathy: A three-group randomized clinical trial. Am. J. Phys. Med. Rehabil. 2014, 93, 1-16. [CrossRef] [PubMed] 
48. Dalal, K.; Maran, V.B.; Pandey, R.M.; Tripathi, M. Determination of efficacy of reflexology in managing patients with diabetic neuropathy: A randomized controlled clinical trial. Evid. Based Complement. Altern. Med. 2014, 2014, 843036. [CrossRef]

49. Song, C.H.; Petrofsky, J.S.; Lee, S.W.; Lee, K.J.; Yim, J.E. Effects of an exercise program on balance and trunk proprioception in older adults with diabetic neuropathies. Diabetes Technol. Ther. 2011, 13, 803-811. [CrossRef]

50. Kanchanasamut, W.; Pensri, P. Effects of weight-bearing exercise on a mini-trampoline on foot mobility, plantar pressure and sensation of diabetic neuropathic feet; a preliminary study. Diabetes Foot Ankle 2017, 8, 1287239. [CrossRef] [PubMed]

51. Boulton, A.J.; Vinik, A.I.; Arezzo, J.C.; Bril, V.; Feldman, E.L.; Freeman, R.; Malik, R.A.; Maser, R.E.; Sosenko, J.M.; Ziegler, D. Diabetic neuropathies: A statement of the American Diabetes Association. Diabetes Care 2005, 28, 956-962. [CrossRef]

52. Amelia, R.; Wahyuni, A.S.; Yunanda, Y. Diabetic neuropathy among type 2 diabetes mellitus patients at amplas primary health care in Medan city. Open Access Maced. J. Med. Sci. 2019, 7, 3400-3403. [CrossRef] [PubMed]

53. Forouhi, N.G.; Wareham, N.J. Epidemiology of diabetes. Medicine 2014, 42, 698-702. [CrossRef] [PubMed]

54. Nagpal, A.S.; Leet, J.; Egan, K.; Garza, R. Diabetic Neuropathy: A Critical, Narrative Review of Published Data from 2019. Curr. Pain Headache Rep. 2021, 25, 15. [CrossRef]

55. Schmid, A.B.; Bland, J.D.P.; Bhat, M.A.; Bennett, D.L.H. The relationship of nerve fibre pathology to sensory function in entrapment neuropathy. Brain 2014, 137, 3186-3199. [CrossRef]

56. Gu, Y.; Dennis, S.M.; Kiernan, M.C.; Harmer, A.R. Aerobic exercise training may improve nerve function in type 2 diabetes and pre-diabetes: A systematic review. Diabetes Metab. Res. Rev. 2019, 35, e3099. [CrossRef] [PubMed]

57. Ites, K.I.; Anderson, E.J.; Cahill, M.L.; Kearney, J.A.; Post, E.C.; Gilchrist, L.S. Balance interventions for Diabetic Peripheral Neuropathy: A systematic review. J. Geriatr. Phys. Ther. 2011, 34, 109-116. [CrossRef]

58. Zhu, G.C.; Tsai, K.L.; Chen, Y.W.; Hung, C.H. Neural mobilization attenuates mechanical allodynia and decreases proinflammatory cytokine concentrations in rats with painful diabetic neuropathy. Phys. Ther. 2018, 98, 214-222. [CrossRef] [PubMed]

59. Larkin, M.E.; Barnie, A.; Braffett, B.H.; Cleary, P.A.; Diminick, L.; Harth, J.; Gatcomb, P.; Golden, E.; Lipps, J.; Lorenzi, G.; et al. Musculoskeletal complications in type 1 diabetes. Diabetes Care 2014, 37, 1863-1869. [CrossRef]

60. Bialosky, J.E.; Bishop, M.D.; Price, D.D.; Robinson, M.E.; Vincent, K.R.; George, S.Z. A randomized sham-controlled trial of a neurodynamic technique in the treatment of carpal tunnel syndrome. J. Orthop. Sports Phys. Ther. 2009, 39, 709-723. [CrossRef] [PubMed]

61. Lima, C.R.; Martins, D.F.; Reed, W.R. Physiological responses induced by manual therapy in animal models: A scoping review. Front. Neurosci. 2020, 14, 430. [CrossRef] 